\title{
Computing the Expected Markov Reward Rates with Stationarity Detection and Relative Error Control
}

\author{
Víctor Suñé
}

the date of receipt and acceptance should be inserted later

\begin{abstract}
By combining in a novel way the randomization method with the stationary detection technique, we develop two new algorithms for the computation of the expected reward rates of finite, irreducible Markov reward models, with control of the relative error. The first algorithm computes the expected transient reward rate and the second one computes the expected averaged reward rate. The algorithms are numerically stable. Further, it is argued that, from the point of view of run-time computational cost, for medium-sized and large Markov reward models, we can expect the algorithms to be better than the only variant of the randomization method that allows to control the relative error and better than the approach that consists in employing iteratively the currently existing algorithms that use the randomization method with stationarity detection but allow to control the absolute error. The performance of the new algorithms is illustrated by means of examples, showing that the algorithms can be not only faster but also more efficient than the alternatives in terms of run-time computational cost in relation to accuracy.
\end{abstract}

Keywords Markov reward model, Markov chain, expected reward rate, relative error, randomization, stationarity detection.

Mathematics Subject Classification (2000) 60J28, 60J22, 65C40.

\section{Introduction}

Consider a finite Markov reward model (MRM) consisting of a finite, irreducible (time homogeneous) continuous-time Markov chain (CTMC) $X=\{X(t), t \geq 0\}$ with infinitesimal generator and a reward rate vector $\mathbf{r}=\left(r_{i}\right)$ whose $i$ th entry has the meaning of "rate" at which reward is earned while $X$ is in state $i$. In this paper, we will be concerned with the computation of the expected transient reward rate at time $t, t>0, \operatorname{ETRR}(t)=\mathrm{E}\left[r_{X(t)}\right]$, and the expected averaged reward rate in the time interval $[0, t], t>0, \operatorname{EARR}(t)=\mathrm{E}\left[(1 / t) \int_{0}^{t} r_{X(\tau)} \mathrm{d} \tau\right]$. To illustrate the usefulness of $\operatorname{ETRR}(t)$ and $\operatorname{EARR}(t)$, consider a CTMC modeling a fault-tolerant system that can be up or down and assume that a reward rate 1 is assigned to the states in which the system is up and a reward rate 0 is assigned to the states in which the system is down. Then, $\operatorname{ETRR}(t)$ would be the availability of

Departament d'Enginyeria Electrònica

Universitat Politècnica de Catalunya

Colom, 1, 08222 Terrassa

Catalonia, Spain

Tel.: +034-93-7398219, Fax: +034-93-7398225

E-mail: victor.sunye@upc.edu 
the system at time $t$, i.e., the probability that the system is up at time $t$, and $\operatorname{EARR}(t)$ would be the expected interval availability at time $t$, i.e., the expected fraction of the time interval $[0, t]$ in which the system is up.

We will assume that all reward rates are nonnegative and, to avoid trivialities, that at least one of them is nonnull. Since $X$ is irreducible, this implies that, for $t>0$, both $\operatorname{ETRR}(t)$ and $\operatorname{EARR}(t)$ are positive. The assumption that there are not negative reward rates is not a true restriction as it can be easily circumvented (Carrasco, 2004).

$\operatorname{Both} \operatorname{ETRR}(t)$ and $\operatorname{EARR}(t)$ can be computed with well-controlled error using the randomization method (also called uniformization) (Grassmann, 1977; Gross and Miller, 1984) and variants (van Moorsel and Sanders, 1994; Sericola, 1999; Carrasco, 2003a, 2004; Suñé and Carrasco, 2005; Sidje et al., 2007). Almost all these variants allow to compute $\operatorname{ETRR}(t)$ and $\operatorname{EARR}(t)$ with control of the absolute error, which is not always satisfactory. The reason is that if $\operatorname{ETRR}(t)$ and $\operatorname{EARR}(t)$ have to be computed with prescribed relative accuracy, then one has to use those algorithms iteratively until the accuracy requirement is fulfilled. To the best of the author's knowledge, the only variants that allow to compute $\operatorname{ETRR}(t)$ and $\operatorname{EARR}(t)$ with control of the relative error are the implementations of the randomization method developed in (Suñé and Carrasco, 2005). However, like most randomization-based methods, when the time $t$ at which $\operatorname{ETRR}(t)$ and $\operatorname{EARR}(t)$ have to be computed is large, those implementations tend to have high run-time computational cost. In the case of finite, irreducible MRMs with infinitesimal generator, the run-time computational cost of the randomization method can be reduced by using the so-called stationarity detection technique. Broadly speaking, the technique consists in detecting when the underlying CTMC is close enough to its stationarity regime so that the computations can be stopped and, therefore, can result in significant reductions of the run-time computational cost when $t$ is large. The stationarity detection technique has already been combined with the randomization method to develop algorithms for the computation of $\operatorname{ETRR}(t)$ and $\operatorname{EARR}(t)$ (Sericola, 1999) which can be much faster than most randomization-based algorithms. ${ }^{1}$ But, those algorithms allow to control the absolute error. Currently, then, to compute $\operatorname{ETRR}(t)$ and $\operatorname{EARR}(t)$ with prescribed relative accuracy, one can use the implementations of the randomization method developed in (Suñe and Carrasco, 2005), which, as previously commented, can be very slow when the time $t$ is large, or else can use iteratively the algorithms developed in (Sericola, 1999), an approach that is not completely satisfactory either because unless a good estimate for $\operatorname{ETRR}(t)$ and $\operatorname{EARR}(t)$ is available, it can be necessary to execute the algorithms twice or more times, thus (partially) offsetting the reduction in run-time computational cost brought up by the stationarity detection technique.

In this paper, by combining in a novel way the randomization method with the stationarity detection technique proposed in (Sericola, 1999), we develop two new algorithms, one for the computation of $\operatorname{ETRR}(t)$ and another for the computation of $\operatorname{EARR}(t)$, with control of the relative error. The algorithms are numerically stable. Compared with the implementations of the randomization method developed in (Suñé and Carrasco, 2005) and the approach that consists in using iteratively the algorithms developed in (Sericola, 1999), the algorithms can be expected to have, for medium-sized and large MRMs, a lower run-time computational cost. Besides, when accuracy is taken into account, the algorithms can be substantially more efficient in the sense of being able to achieve the same accuracy with a much lower run-time computational cost. The rest of the paper is organized as follows. The algorithms are developed in Sections 2 and 3. In Section 4, we discuss the numerical stability and the run-time computational cost of the proposed algorithms. In Section 5, we illustrate the performance of the algorithms and compare them with the alternatives. Finally, in Section 6 we present some conclusions. The Appendix collects the proofs of the theoretical results on which the new algorithms are based.

${ }^{1}$ In (Sericola, 1999), $\operatorname{ETRR}(t)$ and $\operatorname{EARR}(t)$ are referred to as point performability and expected interval perform-ability, respectively. 


\section{Computation of $\operatorname{ETRR}(t)$}

First, we introduce some notations. We will denote by $\boldsymbol{\alpha}$ the initial probability distribution vector of $X$ and by $\mathbf{A}=\left(a_{i, j}\right)$ its infinitesimal generator. The probability of having $j \geq 0$ arrivals in a Poisson distribution with parameter $\lambda>0$ will be denoted $P_{j}(\lambda)=\mathrm{e}^{-\lambda} \lambda^{j} / j$ !. In addition, we will denote by $\mathbf{I}$ the identity matrix, by $\lfloor x\rfloor$ the largest integer nonlarger than $x$, by ${ }^{\mathrm{T}}$ the transpose operator, by $r_{\min }$ the minimal reward rate $\min _{i} r_{i}$, by $r_{\max }$ the maximal reward $\operatorname{rate} \max _{i} r_{i}$, by $\varepsilon$ a positive relative error tolerance, and by $\delta$ a positive quantity $\ll 1$.

Let $\Lambda \geq \max _{i}\left|a_{i, i}\right|$ and $\mathbf{B}=\mathbf{I}+(1 / \Lambda) \mathbf{A}$ and define, for $k \geq 0, \mathbf{c}^{(k)}=\left(c_{i}^{(k)}\right)=\mathbf{B}^{k}\left(\mathbf{r} / r_{\max }\right)$ and $v_{k}=\boldsymbol{\alpha}^{\mathrm{T}} \mathbf{c}^{(\bar{k})}$. Using the well-known randomization result (see, e.g., (Kijima, 1997, Theorem 4.19)), we can write

$$
\begin{aligned}
\operatorname{ETRR}(t) & =\boldsymbol{\alpha}^{\mathrm{T}} \mathrm{e}^{\mathbf{A} t} \mathbf{r} \\
& =\sum_{j=0}^{\infty} \boldsymbol{\alpha}^{\mathrm{T}} \mathbf{B}^{j} \mathrm{e}^{-\Lambda t} \frac{(\Lambda t)^{j}}{j !} \mathbf{r} \\
& =r_{\max } \sum_{j=0}^{\infty} \boldsymbol{\alpha}^{\mathrm{T}} \mathbf{B}^{j} \frac{\mathbf{r}}{r_{\max }} \mathrm{e}^{-\Lambda t} \frac{(\Lambda t)^{j}}{j !} \\
& =r_{\max } \sum_{j=0}^{\infty} \boldsymbol{\alpha}^{\mathrm{T}} \mathbf{c}^{(j)} P_{j}(\Lambda t) \\
& =r_{\max } \sum_{j=0}^{\infty} v_{j} P_{j}(\Lambda t) .
\end{aligned}
$$

Let $\pi$ denote the steady-state probability distribution vector of $X$. If $\Lambda>\max _{i}\left|a_{i, i}\right|$, as $k \rightarrow \infty$ each entry of the vector $\mathbf{c}^{(k)}$ tends to its stationary value $\pi^{\mathrm{T}} \mathbf{r} / r_{\max }$ (Sericola, 1999). Formally, for every state $i$ of $X$,

$$
\lim _{k \rightarrow \infty} \mathbf{c}_{i}^{(k)}=\pi^{\mathrm{T}} \mathbf{r} / r_{\max }
$$

In (Sericola, 1999), the above limit was turned into a practical test for stationarity detection by proving that, given the sequences $\left\{m_{k}=\min _{i} c_{i}^{(k)}\right\}$ and $\left\{M_{k}=\max _{i} c_{i}^{(k)}\right\}$, we have

$$
\left|v_{j}-\frac{m_{k}+M_{k}}{2}\right| \leq \frac{M_{k}-m_{k}}{2}, \quad j \geq k \geq 0,
$$

and, therefore, given $k \geq 0$, the approximation for $\operatorname{ETRR}(t)$ that results from replacing in (1) all $v_{j}$, $j>k$, by $\left(m_{k}+M_{k}\right) / 2$,

$$
\widehat{\operatorname{ETRR}}(t, k)=r_{\max }\left(\sum_{j=0}^{k} v_{j} P_{j}(\Lambda t)+\frac{m_{k}+M_{k}}{2}\left(1-\sum_{j=0}^{k} P_{j}(\Lambda t)\right)\right)
$$

has well-controlled error

$$
|\operatorname{ETRR}(t)-\widehat{\operatorname{ETRR}}(t, k)| \leq r_{\max } \frac{M_{k}-m_{k}}{2}\left(1-\sum_{j=0}^{k} P_{j}(\Lambda t)\right) .
$$

The new algorithm is based on (1), (3). We start by noting that, since, as assumed, $X$ is irreducible and $r_{\max }>0$, we have

$$
M_{k}>0, \quad k \geq 0,
$$


implying $m_{k}+M_{k}>0, k \geq 0$. Therefore, by (4), we have $\widehat{\operatorname{ETRR}}(t, k)>0, t>0, k \geq 0$, and can then define the relative error incurred by approximating $\operatorname{ETRR}(t)$ by $\widehat{\operatorname{ETRR}}(t, k), t>0, k \geq 0$, as

$$
\left|\frac{\operatorname{ETRR}(t)-\widehat{\operatorname{ETRR}}(t, k)}{\widehat{\operatorname{ETRR}}(t, k)}\right|=\frac{|\operatorname{ETRR}(t)-\widehat{\operatorname{ETRR}}(t, k)|}{\widehat{\operatorname{ETRR}}(t, k)} .
$$

Trivially, to make that relative error nonlarger than $\varepsilon$, the index $k$ much be such that $\mid \operatorname{ETRR}(t)-$ $\widehat{\operatorname{ETRR}}(t, k) \mid \leq \varepsilon \widehat{\operatorname{ETRR}}(t, k)$. By $(4),(5)$, to satisfy the previous inequality it is sufficient that

$$
M_{k}-m_{k} \leq\left(2 \frac{\sum_{j=0}^{k} v_{j} P_{j}(\Lambda t)}{1-\sum_{j=0}^{k} P_{j}(\Lambda t)}+m_{k}+M_{k}\right) \varepsilon .
$$

Since, by (2), $\lim _{k \rightarrow \infty}\left(M_{k}-m_{k}\right)=0,(6) M_{k}>0, k \geq 0$, and $\varepsilon>0$, there exist infinitely many indices $k$ satisfying Inequality $(7)$ and $\operatorname{ETRR}(t)$ could be computed with relative error $\leq \varepsilon$ by using (4) with $k$ set to the minimal of those indices. However, that scheme would not be completely satisfactory because of the potential numerical cancellations involved in the computation in (4) of the term $1-\sum_{j=0}^{k} P_{j}(\Lambda t)$ (Inequality (7) can be easily rewritten to avoid the computation of that term) when the sum $\sum_{j=0}^{k} P_{j}(\Lambda t)$ is close to 1 . To avoid those potential numerical cancellations, we will replace $1-\sum_{j=0}^{k} P_{j}(\Lambda t)$ by a lower bound that does not involve significant cancellations and will tighten Inequality (7) to offset the additional error introduced by the bound.

The lower bound is

$$
\left[1-\sum_{j=0}^{k} P_{j}(\Lambda t)\right]^{\mathrm{lb}}= \begin{cases}1-\sum_{j=0}^{k} P_{j}(\Lambda t) & \text { if } \sum_{j=0}^{k} P_{j}(\Lambda t) \leq 0.9 \\ \sum_{j=k+1}^{R_{1}} P_{j}(\Lambda t) & \text { otherwise }\end{cases}
$$

where

$$
R_{1}=\min \left\{r \geq k+1: \frac{\frac{1}{r+3-\Lambda t} \frac{r+3}{r+2}\left(r+2+\frac{\Lambda t}{r+3-\Lambda t}\right) P_{r+1}(\Lambda t)}{\sum_{j=k+1}^{r} P_{j}(\Lambda t)} \leq \delta\right\} .
$$

Proposition 1 below shows that $\left[1-\sum_{j=0}^{k} P_{j}(\Lambda t)\right]^{\mathrm{lb}}$ bounds $1-\sum_{j=0}^{k} P_{j}(\Lambda t)$ from below with a relative error $\leq \delta$.

Proposition 1 Assume $t>0, k \geq 0$, and $\delta>0$. Then, the truncation parameter $R_{1}$ given by (9) is finite and $\left[1-\sum_{j=0}^{k} P_{j}(\Lambda t)\right]^{\mathrm{lb}}$ defined by $(8)$ satisfies

$$
0 \leq \frac{1-\sum_{j=0}^{k} P_{j}(\Lambda t)-\left[1-\sum_{j=0}^{k} P_{j}(\Lambda t)\right]^{\mathrm{lb}}}{1-\sum_{j=0}^{k} P_{j}(\Lambda t)} \leq \delta
$$

That the computation of the lower bound $\left[1-\sum_{j=0}^{k} P_{j}(\Lambda t)\right]^{\mathrm{lb}}$ will not involve significant numerical cancellations can be shown as follows. If $\sum_{j=0}^{k} P_{j}(\Lambda t) \leq 0.9$, then $\left[1-\sum_{j=0}^{k} P_{j}(\Lambda t)\right]^{\mathrm{lb}}=$ $1-\sum_{j=0}^{k} P_{j}(\Lambda t) \geq 0.1$, and, therefore, $\left[1-\sum_{j=0}^{k} P_{j}(\Lambda t)\right]^{\mathrm{lb}}$ can be computed without significant numerical cancellations. If $\sum_{j=0}^{k} P_{j}(\Lambda t)>0.9$, then $\left[1-\sum_{j=0}^{k} P_{j}(\Lambda t)\right]^{\mathrm{lb}}=\sum_{j=k+1}^{R_{1}} P_{j}(\Lambda t)$ and the only subtractions are the ones involved in the computation of the term $r+3-\Lambda t$ in (9). Those subtractions, however, will not involve significant numerical cancellations because, using the fact that the median of a Poisson distribution with parameter $\Lambda t$ is nonsmaller than $\Lambda t-\log 2$ (Choi, 1994), in (9) we will have $k \geq \Lambda t-\log 2$, implying $r+3-\Lambda t \geq k+4-\Lambda t \geq \Lambda t-\log 2+4-\Lambda t>3$.

If in (4) we now replace the term $1-\sum_{j=0}^{k} P_{j}(\Lambda t)$ by the lower bound $\left[1-\sum_{j=0}^{k} P_{j}(\Lambda t)\right]^{1 \mathrm{~b}}$, we obtain the new approximation

$$
\widehat{\operatorname{ETRR}}(t, k)=r_{\max }\left(\sum_{j=0}^{k} v_{j} P_{j}(\Lambda t)+\frac{m_{k}+M_{k}}{2}\left[1-\sum_{j=0}^{k} P_{j}(\Lambda t)\right]^{\mathrm{lb}}\right) .
$$


Let

$$
\varepsilon^{\prime}=\left(1-\delta\left(1+\frac{1}{\varepsilon}\right)\right) \varepsilon=\varepsilon(1-\delta)-\delta
$$

To offset the additional error introduced by the lower bound $\left[1-\sum_{j=0}^{k} P_{j}(\Lambda t)\right]^{\mathrm{lb}}$, we require $k$ in (11) to satisfy

$$
M_{k}-m_{k} \leq 2 \frac{\sum_{j=0}^{k} v_{j} P_{j}(\Lambda t)}{1-\sum_{j=0}^{k} P_{j}(\Lambda t)} \varepsilon+\left(m_{k}+M_{k}\right) \varepsilon^{\prime}
$$

instead of (7). Then, defining

$$
K_{1}=\min \{k \geq 0: \text { Inequality (13) holds }\}
$$

we have the following result.

Proposition 2 Let $t, t^{\prime}, 0<t^{\prime} \leq t$, and assume $0<\delta<1, \varepsilon>\delta /(1-\delta)$. Then, the truncation parameter $K_{1}$ given by (13), (14) is finite and $\widehat{\operatorname{ETRR}}\left(t^{\prime}, K_{1}\right)$ given by (11) with $t$ replaced by $t^{\prime}$ and $k$ replaced by $K_{1}$ satisfies

$$
\left|\frac{\operatorname{ETRR}\left(t^{\prime}\right)-\widehat{\operatorname{ETRR}}\left(t^{\prime}, K_{1}\right)}{\widehat{\operatorname{ETRR}}\left(t^{\prime}, K_{1}\right)}\right| \leq \varepsilon
$$

We are now in a position to describe the proposed algorithm for the computation of $\operatorname{ETRR}(t)$ for a set of $n, n \geq 1$, time points $0<t_{1}<\cdots<t_{n}$. First, we obtain the integer $K_{1}$ using (14) with $t$ set to the largest time point $t_{n}$. To avoid the numerical cancellations potentially involved in the computation of the term $1-\sum_{j=0}^{k} P_{j}(\Lambda t)$ in Inequality (13), that inequality is used in its equivalent form

$$
\begin{aligned}
M_{k}\left(1+\varepsilon^{\prime} \sum_{j=0}^{k} P_{j}(\Lambda t)\right) & +m_{k}\left(1+\varepsilon^{\prime}\right) \sum_{j=0}^{k} P_{j}(\Lambda t) \\
& \leq M_{k}\left(\sum_{j=0}^{k} P_{j}(\Lambda t)+\varepsilon^{\prime}\right)+m_{k}\left(1+\varepsilon^{\prime}\right)+2 \varepsilon \sum_{j=0}^{k} v_{j} P_{j}(\Lambda t)
\end{aligned}
$$

By Proposition 2, the integer $K_{1}$ thus obtained is such that

$$
\left|\frac{\operatorname{ETRR}\left(t_{i}\right)-\widehat{\operatorname{ETRR}}\left(t_{i}, K_{1}\right)}{\widehat{\operatorname{ETRR}}\left(t_{i}, K_{1}\right)}\right| \leq \varepsilon
$$

for all $t_{i}, 0<t_{i} \leq t_{n}$. Therefore, once $K_{1}$ is known, we compute $\widehat{\operatorname{ETRR}}\left(t_{i}, K_{1}\right), 1 \leq i \leq n$, using (8), (9), (11) with $t$ replaced by $t_{i}$ and $k$ replaced by $K_{1}$. Finally, since $\operatorname{ETRR}(\infty)=\bar{\pi}^{\mathrm{T}} \mathbf{r}$ and (Sericola, 1999)

$$
m_{k} \leq \pi^{\mathrm{T}} \mathbf{r} / r_{\max } \leq M_{k}, \quad k \geq 0
$$

we also compute the bounds $r_{\max } m_{K_{1}} \leq \operatorname{ETRR}(\infty) \leq r_{\max } M_{K_{1}}$. A detailed description of the algorithm follows. 


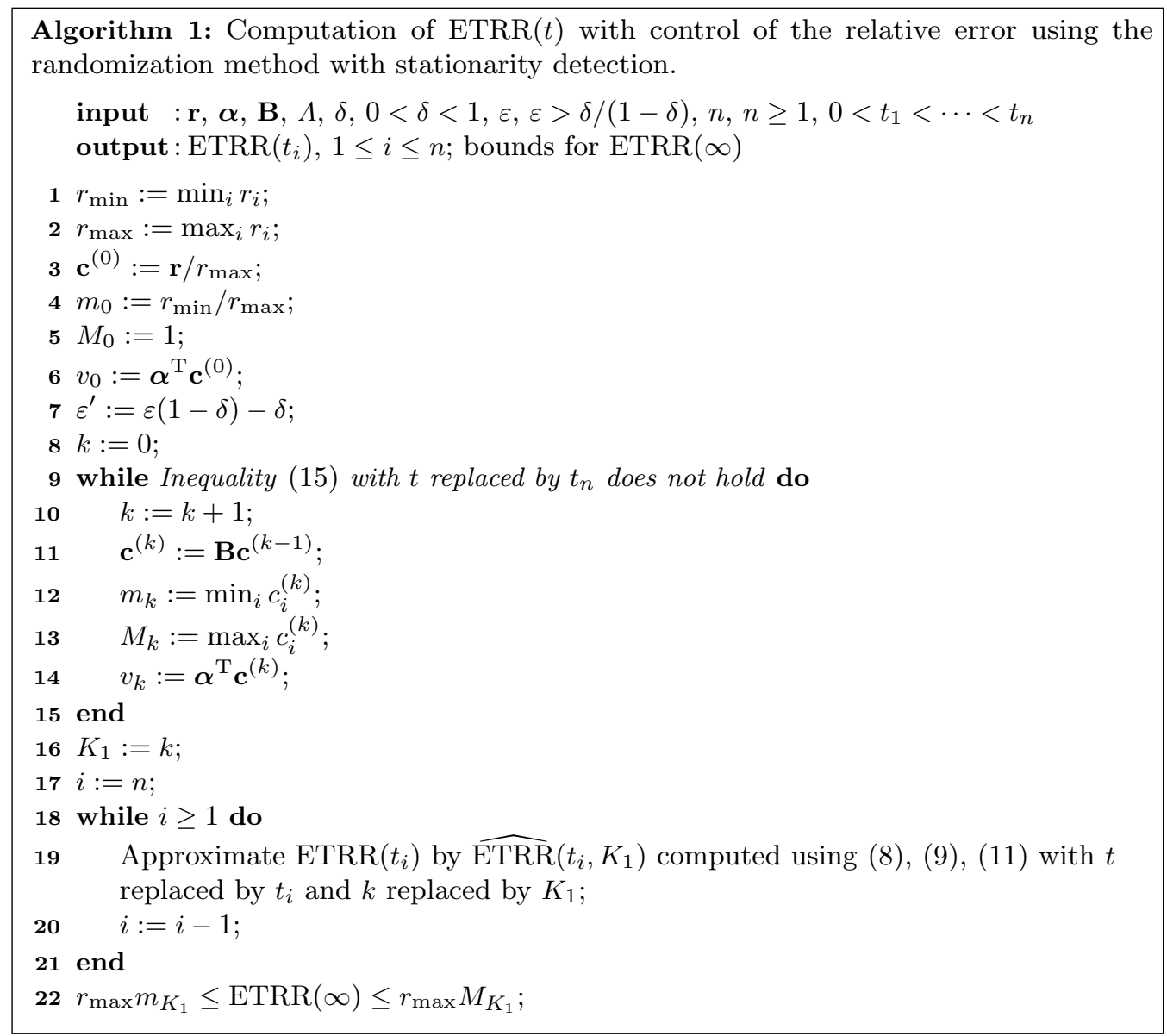

Since the parameter $\delta$ in Algorithm 1 must be positive and the larger it is, making $\varepsilon^{\prime}$ smaller, the larger $K_{1}$ can be, in practice one will use the algorithm with $\delta$ set to a positive quantity $\ll 1$, e.g., some multiple of the machine epsilon.

To conclude this section, we note that the bounds $r_{\max } m_{K_{1}}$ and $r_{\max } M_{K_{1}}$ computed by the proposed algorithm can be used to approximate $\operatorname{ETRR}(\infty)$ by $\widehat{\operatorname{ETRR}}\left(\infty, K_{1}\right)=r_{\max }\left(m_{K_{1}}+M_{K_{1}}\right) / 2$. Since (Sericola, 1999) $\left|\operatorname{ETRR}(\infty)-r_{\max }\left(m_{k}+M_{k}\right) / 2\right| \leq r_{\max }\left(M_{k}-m_{k}\right) / 2, k \geq 0$, the incurred relative error will satisfy

$$
\left|\frac{\operatorname{ETRR}(\infty)-\widehat{\operatorname{ETRR}}\left(\infty, K_{1}\right)}{\widehat{\operatorname{ETRR}}\left(\infty, K_{1}\right)}\right| \leq \frac{M_{K_{1}}-m_{K_{1}}}{M_{K_{1}}+m_{K_{1}}}
$$

For large enough $\Lambda t_{n}$, we can expect that error to be close to $\varepsilon$. Indeed, it is easy to check that, for a fixed value of $k$, the function $\left(\sum_{j=0}^{k} v_{j} P_{j}(\Lambda t)\right) /\left(1-\sum_{j=0}^{k} P_{j}(\Lambda t)\right)$ decreases to 0 as $\Lambda t \rightarrow \infty$. Therefore, by (13), (14), for large enough $\Lambda t_{n}$, the parameter $K_{1}$ will be almost independent of $\Lambda t_{n}$, satisfying $M_{K_{1}}-m_{K_{1}} \approx\left(m_{K_{1}}+M_{K_{1}}\right) \varepsilon^{\prime}$ and, consequently, we will have

$$
\left|\frac{\operatorname{ETRR}(\infty)-\widehat{\operatorname{ETRR}}\left(\infty, K_{1}\right)}{\widehat{\operatorname{ETRR}}\left(\infty, K_{1}\right)}\right| \leq \frac{M_{K_{1}}-m_{K_{1}}}{M_{K_{1}}+m_{K_{1}}} \approx \varepsilon^{\prime}<\varepsilon .
$$




\section{Computation of $\operatorname{EARR}(t)$}

Using $\operatorname{EARR}(t)=(1 / t) \int_{0}^{t} \operatorname{ETRR}(\tau) \mathrm{d} \tau,(1)$, and $\int_{0}^{t} P_{l}(\Lambda \tau) \mathrm{d} \tau=(1 / \Lambda) \sum_{j=l+1}^{\infty} P_{j}(\Lambda t)$, we obtain the well-known result

$$
\begin{aligned}
\operatorname{EARR}(t) & =\frac{1}{t} \int_{0}^{t} \operatorname{ETRR}(\tau) \mathrm{d} \tau \\
& =r_{\max } \sum_{l=0}^{\infty} \frac{1}{t} \int_{0}^{t} v_{l} P_{l}(\Lambda \tau) \mathrm{d} \tau \\
& =r_{\max } \sum_{l=0}^{\infty} v_{l} \frac{1}{\Lambda t} \sum_{j=l+1}^{\infty} P_{j}(\Lambda t) \\
& =r_{\max } \sum_{l=0}^{\infty} v_{l} \sum_{j=l}^{\infty} \frac{1}{j+1} P_{j}(\Lambda t) \\
& =r_{\max } \sum_{j=0}^{\infty} \frac{1}{j+1} \sum_{l=0}^{j} v_{l} P_{j}(\Lambda t) \\
& =r_{\max } \sum_{j=0}^{\infty} w_{j} P_{j}(\Lambda t),
\end{aligned}
$$

where

$$
w_{j}=\frac{1}{j+1} \sum_{l=0}^{j} v_{l}
$$

Again, as in (Sericola, 1999), given some $k, k \geq 0$, we will replace all $v_{j}, j>k$, in (18) by $\left(m_{k}+M_{k}\right) / 2$, obtaining the approximation for $\operatorname{EARR}(t)$,

$$
\widehat{\operatorname{EARR}}(t, k)=r_{\max }\left(\sum_{j=0}^{k} w_{j} P_{j}(\Lambda t)+\sum_{j=k+1}^{\infty} \frac{1}{j+1}\left(\sum_{l=0}^{k} v_{l}+\sum_{l=k+1}^{j} \frac{m_{k}+M_{k}}{2}\right) P_{j}(\Lambda t)\right) .
$$

Since, by $(6), m_{k}+M_{k}>0, k \geq 0$, we have $\widehat{\operatorname{EARR}}(t, k)>0, t>0, k \geq 0$, and can then define the relative error incurred by approximating $\operatorname{EARR}(t)$ by $\widehat{\operatorname{EARR}}(t, k)$ as

$$
\left|\frac{\operatorname{EARR}(t)-\widehat{\operatorname{EARR}}(t, k)}{\widehat{\operatorname{EARR}}(t, k)}\right|=\frac{|\operatorname{EARR}(t)-\widehat{\operatorname{EARR}}(t, k)|}{\widehat{\operatorname{EARR}}(t, k)} .
$$

For that relative error to be nonlarger than $\varepsilon$, the index $k$ must be such that

$$
|\operatorname{EARR}(t)-\widehat{\operatorname{EARR}}(t, k)| \leq \varepsilon \widehat{\operatorname{EARR}}(t, k) .
$$

For that inequality to be useful, we need computable expressions for $\widehat{\operatorname{EARR}}(t, k)$ and $\mid \operatorname{EARR}(t)-$ $\widehat{\operatorname{EARR}}(t, k) \mid$. Let us start with $\widehat{\operatorname{EARR}}(t, k)$. By combining $(19),(20)$, and

$$
\begin{aligned}
\sum_{j=k+1}^{\infty} \frac{j-k}{j+1} P_{j}(\Lambda t) & =\sum_{j=k+1}^{\infty} \frac{j+1-(k+1)}{j+1} P_{j}(\Lambda t) \\
& =\sum_{j=k+1}^{\infty} P_{j}(\Lambda t)-\frac{k+1}{\Lambda t} \sum_{j=k+2}^{\infty} P_{j}(\Lambda t)
\end{aligned}
$$




$$
=P_{k+1}(\Lambda t)+\frac{\Lambda t-(k+1)}{\Lambda t}\left(1-\sum_{j=0}^{k+1} P_{j}(\Lambda t)\right)
$$

we obtain

$$
\begin{aligned}
\widehat{\operatorname{EARR}}(t, k)= & r_{\max }\left(\sum_{j=0}^{k} w_{j} P_{j}(\Lambda t)+\sum_{j=k+1}^{\infty}\left(\frac{k+1}{j+1} w_{k}+\frac{j-k}{j+1} \frac{m_{k}+M_{k}}{2}\right) P_{j}(\Lambda t)\right) \\
= & r_{\max }\left(\sum_{j=0}^{k} w_{j} P_{j}(\Lambda t)+\frac{k+1}{\Lambda t} w_{k} \sum_{j=k+2}^{\infty} P_{j}(\Lambda t)+\frac{m_{k}+M_{k}}{2} \sum_{j=k+1}^{\infty} \frac{j-k}{j+1} P_{j}(\Lambda t)\right) \\
= & r_{\max }\left(\sum_{j=0}^{k} w_{j} P_{j}(\Lambda t)+\frac{k+1}{\Lambda t} w_{k}\left(1-\sum_{j=0}^{k+1} P_{j}(\Lambda t)\right)+\frac{m_{k}+M_{k}}{2}\left(P_{k+1}(\Lambda t)\right.\right. \\
& \left.+\frac{\Lambda t-(k+1)}{\Lambda t}\left(1-\sum_{j=0}^{k+1} P_{j}(\Lambda t)\right)\right) .
\end{aligned}
$$

With regard to $|\operatorname{EARR}(t)-\widehat{\operatorname{EARR}}(t, k)|$, using (3), (18), (19), (20), and (22), we get

$$
\begin{aligned}
\mid \operatorname{EARR}(t)- & \widehat{\operatorname{EARR}}(t, k) \mid \\
= & \mid r_{\max }\left(\sum_{j=0}^{k} w_{j} P_{j}(\Lambda t)+\sum_{j=k+1}^{\infty} \frac{1}{j+1}\left(\sum_{l=0}^{k} v_{l}+\sum_{l=k+1}^{j} v_{l}\right) P_{j}(\Lambda t)\right) \\
& -r_{\max }\left(\sum_{j=0}^{k} w_{j} P_{j}(\Lambda t)+\sum_{j=k+1}^{\infty} \frac{1}{j+1}\left(\sum_{l=0}^{k} v_{l}+\sum_{l=k+1}^{j} \frac{m_{k}+M_{k}}{2}\right) P_{j}(\Lambda t)\right) \mid \\
= & r_{\max }\left|\sum_{j=k+1}^{\infty} \frac{1}{j+1} \sum_{l=k+1}^{j}\left(v_{l}-\frac{m_{k}+M_{k}}{2}\right) P_{j}(\Lambda t)\right| \\
\leq & r_{\max } \sum_{j=k+1}^{\infty} \frac{1}{j+1} \sum_{l=k+1}^{j}\left|v_{l}-\frac{m_{k}+M_{k}}{2}\right| P_{j}(\Lambda t) \\
\leq & r_{\max } \sum_{j=k+1}^{\infty} \frac{1}{j+1} \sum_{l=k+1}^{j} \frac{M_{k}-m_{k}}{2} P_{j}(\Lambda t) \\
= & r_{\max } \frac{M_{k}-m_{k}}{2} \sum_{j=k+1}^{\infty} \frac{j-k}{j+1} P_{j}(\Lambda t) \\
= & r_{\max } \frac{M_{k}-m_{k}}{2}\left(P_{k+1}(\Lambda t)+\frac{\Lambda t-(k+1)}{\Lambda t}\left(1-\sum_{j=0}^{k+1} P_{j}(\Lambda t)\right)\right) .
\end{aligned}
$$

Then, using (23), (24), it is easily seen that Inequality (21) holds for any index $k$ satisfying

$$
M_{k}-m_{k} \leq\left(2 \frac{\sum_{j=0}^{k} w_{j} P_{j}(\Lambda t)+\frac{k+1}{\Lambda t} w_{k}\left(1-\sum_{j=0}^{k+1} P_{j}(\Lambda t)\right)}{P_{k+1}(\Lambda t)+\frac{\Lambda t-(k+1)}{\Lambda t}\left(1-\sum_{j=0}^{k+1} P_{j}(\Lambda t)\right)}+m_{k}+M_{k}\right) \varepsilon .
$$

However, in order to obtain a simpler algorithm, we will consider the inequality

$$
M_{k}-m_{k} \leq\left(2 \frac{\sum_{j=0}^{k} w_{j} P_{j}(\Lambda t)}{P_{k+1}(\Lambda t)+\frac{\Lambda t-(k+1)}{\Lambda t}\left(1-\sum_{j=0}^{k+1} P_{j}(\Lambda t)\right)}+m_{k}+M_{k}\right) \varepsilon
$$


which, since $1-\sum_{j=0}^{k+1} P_{j}(\Lambda t)>0$, is more restrictive than $(25)$.

Since, by $(2), \lim _{k \rightarrow \infty}\left(M_{k}-m_{k}\right)=0,(6) M_{k}>0$, and $\varepsilon>0$, there are infinitely many indices $k$ satisfying $(26)$ and $\operatorname{EARR}(t)$ could be computed with relative error $\leq \varepsilon$ by using (23) with $k$ set to the minimal of those indices. This, however, could be problematic because of the numerical cancellations potentially involved in the computation of the terms $1-\sum_{j=0}^{k+1} P_{j}(\Lambda t)$ and $P_{k+1}(\Lambda t)+((\Lambda t-(k+1)) /(\Lambda t))\left(1-\sum_{j=0}^{k+1} P_{j}(\Lambda t)\right)$ (Inequality $(26)$ can be easily rewritten to avoid the computation of the latter term). To avoid those potential numerical cancellations, we will replace those terms by appropriate lower bounds not involving significant cancellations and will modify Inequality (26) appropriately.

The term $1-\sum_{j=0}^{k+1} P_{j}(\Lambda t)$ will be replaced by

$$
\left[1-\sum_{j=0}^{k+1} P_{j}(\Lambda t)\right]^{\mathrm{lb}}= \begin{cases}1-\sum_{j=0}^{k+1} P_{j}(\Lambda t) & \text { if } \sum_{j=0}^{k+1} P_{j}(\Lambda t) \leq 0.9 \\ \sum_{j=k+2}^{R_{2}} P_{j}(\Lambda t) & \text { otherwise }\end{cases}
$$

where

$$
R_{2}=\min \left\{r \geq k+2: \frac{\frac{1}{r+3-\Lambda t} \frac{r+3}{r+2}\left(r+2+\frac{\Lambda t}{r+3-\Lambda t}\right) P_{r+1}(\Lambda t)}{\sum_{j=k+2}^{r} P_{j}(\Lambda t)} \leq \delta\right\}
$$

and, recalling $(22)$, the term $P_{k+1}(\Lambda t)+((\Lambda t-(k+1)) /(\Lambda t))\left(1-\sum_{j=0}^{k+1} P_{j}(\Lambda t)\right)=\sum_{j=k+1}^{\infty}((j-$ $k) /(j+1)) P_{j}(\Lambda t)$ will be replaced by

$$
\left[\sum_{j=k+1}^{\infty} \frac{j-k}{j+1} P_{j}(\Lambda t)\right]^{\mathrm{lb}}= \begin{cases}P_{k+1}(\Lambda t) & \text { if } k+1 \leq \Lambda t \text { or else } 1- \\
+\frac{\Lambda t-(k+1)}{\Lambda t}\left(1-\sum_{j=0}^{k+1} P_{j}(\Lambda t)\right) & \begin{array}{l}
0 \frac{\Lambda t}{k+1-\Lambda t} P_{k+1}(\Lambda t) \leq \\
\sum_{j=0}^{k+1} P_{j}(\Lambda t) \leq 0.9 \\
\sum_{j=k+1}^{R_{3}} \frac{j-k}{j+1} P_{j}(\Lambda t)
\end{array} \\
\text { otherwise }\end{cases}
$$

where

$$
R_{3}=\min \left\{r \geq k+1: \frac{\frac{1}{r+3-\Lambda t} \frac{r+3}{r+2}\left(r+1-k+\frac{\Lambda t}{r+3-\Lambda t}\right) P_{r+1}(\Lambda t)}{\sum_{j=k+1}^{r} \frac{j-k}{j+1} P_{j}(\Lambda t)} \leq \delta\right\} .
$$

The factor $\Lambda t-(k+1)$ in $(29)$ will be computed accurately by casting it as the dot product $(\Lambda, k+1)(t,-1)$ and computing that dot product using Algorithm 5.3 in (Ogita et al., 2005). By Proposition 3 below, $\left[1-\sum_{j=0}^{k+1} P_{j}(\Lambda t)\right]^{\text {lb }}$ bounds $1-\sum_{j=0}^{k+1} P_{j}(\Lambda t)$ from below with a relative error $\leq \delta$ and $\left[\sum_{j=k+1}^{\infty}((j-k) /(j+1)) P_{j}(\Lambda t)\right]^{\mathrm{lb}}$ bounds $P_{k+1}(\Lambda t)+((\Lambda t-(k+1)) /(\Lambda t))\left(1-\sum_{j=0}^{k+1} P_{j}(\Lambda t)\right)$ from below with a relative error $\leq \delta$.

Proposition 3 Let $t>0, k \geq 0$, and $\delta>0$. Then, the truncation parameters $R_{2}$ and $R_{3}$ given by, respectively, (28) and (30) are finite, $\left[1-\sum_{j=0}^{k+1} P_{j}(\Lambda t)\right]^{\mathrm{lb}}$ defined by $(27)$ satisfies

$$
0 \leq \frac{1-\sum_{j=0}^{k+1} P_{j}(\Lambda t)-\left[1-\sum_{j=0}^{k+1} P_{j}(\Lambda t)\right]^{\mathrm{lb}}}{1-\sum_{j=0}^{k+1} P_{j}(\Lambda t)} \leq \delta
$$

and $\left[\sum_{j=k+1}^{\infty}((j-k) /(j+1)) P_{j}(\Lambda t)\right]^{\mathrm{lb}}$ defined by $(29)$ satisfies

$$
0 \leq \frac{P_{k+1}(\Lambda t)+\frac{\Lambda t-(k+1)}{\Lambda t}\left(1-\sum_{j=0}^{k+1} P_{j}(\Lambda t)\right)-\left[\sum_{j=k+1}^{\infty} \frac{j-k}{j+1} P_{j}(\Lambda t)\right]^{\mathrm{lb}}}{P_{k+1}(\Lambda t)+\frac{\Lambda t-(k+1)}{\Lambda t}\left(1-\sum_{j=0}^{k+1} P_{j}(\Lambda t)\right)} \leq \delta .
$$


That the computation of the lower bound $\left[1-\sum_{j=0}^{k+1} P_{j}(\Lambda t)\right]^{\text {lb }}$ will not involve significant numerical cancellations can be shown similarly as it has been done for the computation of the lower bound (8) $\left[1-\sum_{j=0}^{k} P_{j}(\Lambda t)\right]^{\mathrm{lb}}$. To show that the computation of the lower bound $\left[\sum_{j=k+1}^{\infty}((j-\right.$ $\left.k) /(j+1)) P_{j}(\Lambda t)\right]^{1 \mathrm{~b}}$ will not involve significant numerical cancellations, we will consider three cases separately: a) $k+1 \leq \Lambda t$; b) $k+1>\Lambda t, 1-(0.9 \Lambda t /(k+1-\Lambda t)) P_{k+1}(\Lambda t) \leq \sum_{j=0}^{k+1} P_{j}(\Lambda t) \leq 0.9$; and c $)$ otherwise, i.e., $k+1>\Lambda t$, and $1-(0.9 \Lambda t /(k+1-\Lambda t)) P_{k+1}(\Lambda t)>\sum_{j=0}^{k+1} P_{j}(\Lambda t)$ or $\sum_{j=0}^{k+1} P_{j}(\Lambda t)>0.9$. In case a), which can only happen if $\Lambda t \geq 1$, the only possible source of numerical cancellations is the computation of the term $1-\sum_{j=0}^{k+1} P_{j}(\Lambda t)$ in $(29)$. (We recall that the factor $\Lambda t-(k+1)$ will be computed accurately.) Using the fact that the median of a Poisson distribution with parameter $\Lambda t$ is nonsmaller than $\Lambda t-\log 2$ (Choi, 1994) and noting that, trivially, $\lfloor\Lambda t\rfloor-1<\lfloor\Lambda t\rfloor-\log 2 \leq \Lambda t-\log 2$, we will have $\sum_{j=0}^{\lfloor\Lambda t\rfloor-1} P_{j}(\Lambda t)<0.5$ and, therefore, for $k+1 \leq \Lambda t$, or, equivalently, $k+1 \leq\lfloor\Lambda t\rfloor$,

$$
1-\sum_{j=0}^{k+1} P_{j}(\Lambda t) \geq 1-\sum_{j=0}^{\lfloor\Lambda t\rfloor} P_{j}(\Lambda t)=1-\sum_{j=0}^{\lfloor\Lambda t\rfloor-1} P_{j}(\Lambda t)-P_{\lfloor\Lambda t\rfloor}(\Lambda t)>0.5-P_{\lfloor\Lambda t\rfloor}(\Lambda t) .
$$

Now, since $\Lambda t \geq 1$, either $\lfloor\Lambda t\rfloor=1$ or $\lfloor\Lambda t\rfloor \geq 2$. If $\lfloor\Lambda t\rfloor=1, P_{\lfloor\Lambda t\rfloor}(\Lambda t)=P_{1}(\Lambda t)=\Lambda t \mathrm{e}^{-\Lambda t}$, which reaches its maximum at $\Lambda t=1$ and is therefore nonlarger than $P_{1}(1)=\mathrm{e}^{-1}<0.4$, implying, by (33), $1-\sum_{j=0}^{k+1} P_{j}(\Lambda t)>0.5-0.4=0.1$. If $\lfloor\Lambda t\rfloor \geq 2$, we have (Glynn, 1987) $P_{\lfloor\Lambda t\rfloor}(\Lambda t) \leq 1 / \sqrt{2 \pi\lfloor\Lambda t\rfloor}$, which, for $\lfloor\Lambda t\rfloor \geq 2$, is $<0.3$, implying, by $(33), 1-\sum_{j=0}^{k+1} P_{j}(\Lambda t)>0.5-0.3=0.2$. This shows that, in case a), the term $1-\sum_{j=0}^{k+1} P_{j}(\Lambda t)$ and, therefore, the lower bound $\left[\sum_{j=k+1}^{\infty}((j-k) /(j+\right.$ 1)) $\left.P_{j}(\Lambda t)\right]^{\mathrm{lb}}$, can be computed without significant numerical cancellations. In case b), in which $k+1>\Lambda t, 1-(0.9 \Lambda t /(k+1-\Lambda t)) P_{k+1}(\Lambda t) \leq \sum_{j=0}^{k+1} P_{j}(\Lambda t)$, and $\sum_{j=0}^{k+1} P_{j}(\Lambda t) \leq 0.9$, we will have $1-\sum_{j=0}^{k+1} P_{j}(\Lambda t) \geq 0.1$. This implies that the only possible source of significant numerical cancellations lies in the subtraction of the quantity $((\Lambda t-(k+1)) /(\Lambda t))\left(1-\sum_{j=0}^{k+1} P_{j}(\Lambda t)\right)$ from the probability $P_{k+1}(\Lambda t)$ in $(29)$. But, it can be seen that $1-(0.9 \Lambda t /(k+1-\Lambda t)) P_{k+1}(\Lambda t) \leq \sum_{j=0}^{k+1} P_{j}(\Lambda t)$ implies $P_{k+1}(\Lambda t)+((\Lambda t-(k+1)) /(\Lambda t))\left(1-\sum_{j=0}^{k+1} P_{j}(\Lambda t) \geq 0.1 P_{k+1}(\Lambda t)\right.$. Consequently, in case $\mathrm{b})$, the lower bound $\left[\sum_{j=k+1}^{\infty}((j-k) /(j+1)) P_{j}(\Lambda t)\right]^{\text {lb }}$ can also be computed without significant numerical cancellations. It remains to discuss case c). In that case, $\left[\sum_{j=k+1}^{\infty}((j-k) /(j+1)) P_{j}(\Lambda t)\right]^{\mathrm{lb}}=$ $\sum_{j=k+1}^{R_{3}}((j-k) /(j+1)) P_{j}(\Lambda t)$ and the only subtractions will be the ones involved in the computation of the term $r+3-\Lambda t$ in (30). (The term $r+1-k$ involves only integers and can be computed exactly.) But, those subtractions will not involve significant numerical cancellations because we will have $r+3-\Lambda t \geq k+1+3-\Lambda t>3$. This concludes the justification that the computation of the lower bound $\left[\sum_{j=k+1}^{\infty}((j-k) /(j+1)) P_{j}(\Lambda t)\right]^{\mathrm{lb}}$ will not involve significant numerical cancellations.

Having defined the bounds, in $(23)$ we replace $1-\sum_{j=0}^{k+1} P_{j}(\Lambda t)$ by $\left[1-\sum_{j=0}^{k+1} P_{j}(\Lambda t)\right]^{\mathrm{lb}}$ and $P_{k+1}(\Lambda t)+((\Lambda t-(k+1)) /(\Lambda t))\left(1-\sum_{j=0}^{k+1} P_{j}(\Lambda t)\right)$ by $\left[\sum_{j=k+1}^{\infty}((j-k) /(j+1)) P_{j}(\Lambda t)\right]^{\mathrm{lb}}$, obtaining the new approximation

$$
\begin{aligned}
\widehat{\operatorname{EARR}}(t, k)= & r_{\max } \sum_{j=0}^{k} w_{j} P_{j}(\Lambda t)+r_{\max } \frac{k+1}{\Lambda t} w_{k}\left[1-\sum_{j=0}^{k+1} P_{j}(\Lambda t)\right]^{\mathrm{lb}} \\
& +r_{\max } \frac{m_{k}+M_{k}}{2}\left[\sum_{j=k+1}^{\infty} \frac{j-k}{j+1} P_{j}(\Lambda t)\right]^{\mathrm{lb}},
\end{aligned}
$$

and, to offset the additional errors introduced by the bounds, require $k$ to satisfy

$$
M_{k}-m_{k} \leq 2 \frac{\sum_{j=0}^{k} w_{j} P_{j}(\Lambda t)}{P_{k+1}(\Lambda t)+\frac{\Lambda t-(k+1)}{\Lambda t}\left(1-\sum_{j=0}^{k+1} P_{j}(\Lambda t)\right)} \varepsilon+\left(m_{k}+M_{k}\right) \varepsilon^{\prime}
$$


instead of (26), where $\varepsilon^{\prime}$ is given by (12). Then, defining

$$
K_{2}=\min \{k \geq 0: \text { Inequality (35) holds }\},
$$

we have the following result.

Proposition 4 Let $0<t^{\prime} \leq t$ and assume $0<\delta<1, \varepsilon>\delta /(1-\delta)$. Then, the parameter $K_{2}$ given by (35), (36) is finite and $\widehat{\operatorname{EARR}}\left(t^{\prime}, K_{2}\right)$ given by (34) with $t$ replaced by $t^{\prime}$ and $k$ replaced by $K_{2}$ satisfies

$$
\left|\frac{\operatorname{EARR}\left(t^{\prime}\right)-\widehat{\operatorname{EARR}}\left(t^{\prime}, K_{2}\right)}{\widehat{\operatorname{EARR}}\left(t^{\prime}, K_{2}\right)}\right| \leq \varepsilon .
$$

We are now in a position to describe the proposed algorithm for the computation of $\operatorname{EARR}(t)$ for a set of $n, n \geq 1$, time points $0<t_{1}<\cdots<t_{n}$. First, we obtain the integer $K_{2}$ using (36) with $t$ set to the largest time point $t_{n}$, where the weights $w_{k}, k \geq 0$, are computed using $w_{0}=v_{0}$ and $w_{k}=(1 /(k+1))\left(k w_{k-1}+v_{k}\right), k \geq 1$. To avoid the numerical cancellations potentially involved in the computation of the term $P_{k+1}(\Lambda t)+((\Lambda t-(k+1)) /(\Lambda t))\left(1-\sum_{j=0}^{k+1} P_{j}(\Lambda t)\right)$ in Inequality $(35)$, that inequality is used in its equivalent form

$$
\begin{aligned}
M_{k}\left(P_{k+1}(\Lambda t)+1+\right. & \left.\frac{k+1}{\Lambda t}\left(\sum_{j=0}^{k+1} P_{j}(\Lambda t)+\varepsilon^{\prime}\right)+\varepsilon^{\prime} \sum_{j=0}^{k+1} P_{j}(\Lambda t)\right)+m_{k}\left(\sum_{j=0}^{k+1} P_{j}(\Lambda t)+\frac{k+1}{\Lambda t}\right)\left(1+\varepsilon^{\prime}\right) \\
\leq & M_{k}\left(\sum_{j=0}^{k+1} P_{j}(\Lambda t)+\frac{k+1}{\Lambda t}+\varepsilon^{\prime}\left(P_{k+1}(\Lambda t)+1+\frac{k+1}{\Lambda t} \sum_{j=0}^{k+1} P_{j}(\Lambda t)\right)\right) \\
& +m_{k}\left(1+P_{k+1}(\Lambda t)+\frac{k+1}{\Lambda t} \sum_{j=0}^{k+1} P_{j}(\Lambda t)\right)\left(1+\varepsilon^{\prime}\right)+2 \sum_{j=0}^{k} w_{j} P_{j}(\Lambda t) \varepsilon
\end{aligned}
$$

By Proposition 4, the integer $K_{2}$ thus obtained is such that

$$
\left|\frac{\operatorname{EARR}\left(t_{i}\right)-\widehat{\operatorname{EARR}}\left(t_{i}, K_{2}\right)}{\widehat{\operatorname{EARR}}\left(t_{i}, K_{2}\right)}\right| \leq \varepsilon
$$

for all $t_{i}, 0<t_{i} \leq t_{n}$. Therefore, once $K_{2}$ is known, we compute $\widehat{\operatorname{EARR}}\left(t_{i}, K_{2}\right), 1 \leq i \leq n$, using $(27)$, (28), (29), (30), (34) with $t$ replaced by $t_{i}$ and $k$ replaced by $K_{2}$. Finally, since $\operatorname{EARR}(\infty)=\pi^{\mathrm{T}} \mathbf{r}$ and (16), we also compute the bounds $r_{\max } m_{K_{2}} \leq \operatorname{EARR}(\infty) \leq r_{\max } M_{K_{2}}$. A detailed description of the algorithm follows.

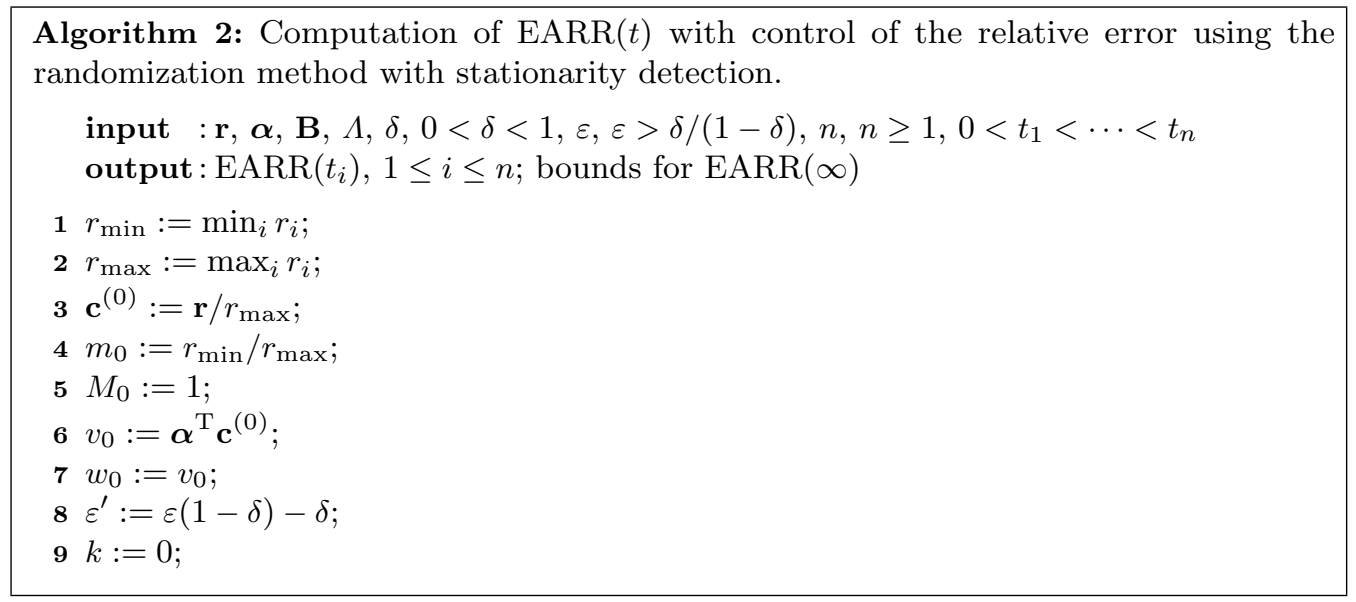




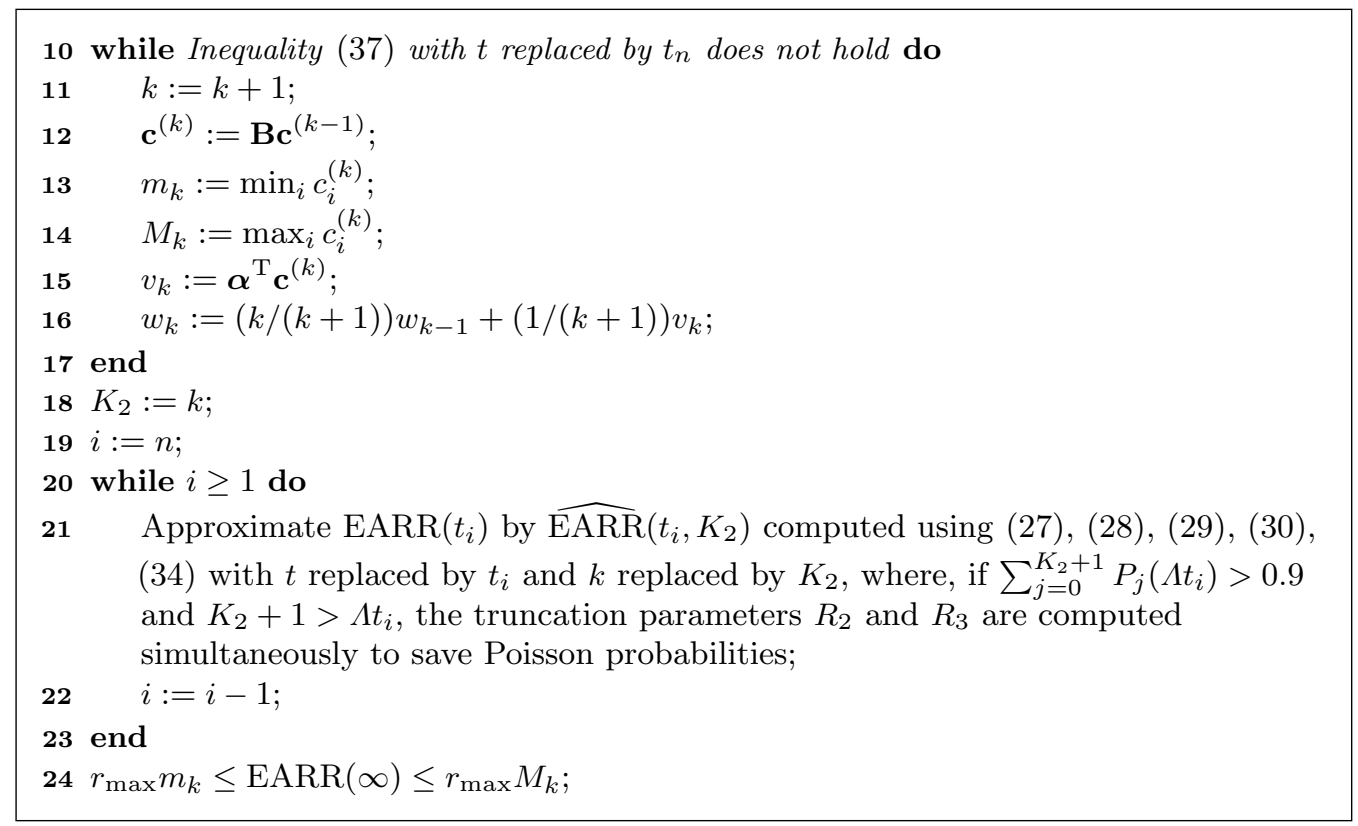

In practice, Algorithm 2 will be used with $\delta$ set to a positive quantity $\ll 1$, e.g., some multiple of the machine epsilon.

To conclude this section, we note that the bounds $r_{\max } m_{K_{2}}$ and $r_{\max } M_{K_{2}}$ computed by Algorithm 2 can be used to approximate $\operatorname{EARR}(\infty)$ by $\widehat{\operatorname{EARR}}\left(\infty, K_{2}\right)=r_{\max }\left(m_{K_{2}}+M_{K_{2}}\right) / 2$. Since $\operatorname{EARR}(\infty)=\operatorname{ETRR}(\infty), \widehat{\operatorname{EARR}}\left(\infty, K_{2}\right)=\widehat{\operatorname{ETRR}}\left(\infty, K_{2}\right)$, and $(17)$, the relative error incurred by that approximation will satisfy

$$
\left|\frac{\operatorname{EARR}(\infty)-\widehat{\operatorname{EARR}}\left(\infty, K_{2}\right)}{\widehat{\operatorname{EARR}}\left(\infty, K_{2}\right)}\right| \leq \frac{M_{K_{2}}-m_{K_{2}}}{M_{K_{2}}+m_{K_{2}}}
$$

For large enough $\Lambda t_{n}$, we can expect that error to be close to $\varepsilon$. Indeed, by (22) we have

$$
\frac{\sum_{j=0}^{k} w_{j} P_{j}(\Lambda t)}{P_{k+1}(\Lambda t)+\frac{\Lambda t-(k+1)}{\Lambda t}\left(1-\sum_{j=0}^{k+1} P_{j}(\Lambda t)\right)}=\frac{\sum_{j=0}^{k} w_{j} P_{j}(\Lambda t)}{\sum_{j=k+1}^{\infty} \frac{j-k}{j+1} P_{j}(\Lambda t)},
$$

a function that decreases to 0 as $\Lambda t \rightarrow \infty$. Therefore, by (35), (36), for large enough $\Lambda t_{n}$, the parameter $K_{2}$ will be almost independent of $\Lambda t_{n}$, satisfying $M_{K_{2}}-m_{K_{2}} \approx\left(m_{K_{2}}+M_{K_{2}}\right) \varepsilon^{\prime}$ and, then, by (38), we will have

$$
\left|\frac{\operatorname{EARR}(\infty)-\widehat{\operatorname{EARR}}\left(\infty, K_{2}\right)}{\widehat{\operatorname{EARR}}\left(\infty, K_{2}\right)}\right| \leq \frac{M_{K_{2}}-m_{K_{2}}}{M_{K_{2}}+m_{K_{2}}} \approx \varepsilon^{\prime}<\varepsilon .
$$

\section{Numerical Stability and Run-Time Computational Cost}

In this section, we will analyze the numerical stability and run-time computational cost of the proposed algorithms. We will also argue that, for medium-sized and large MRMs, we can expect the run-time computational cost of the proposed algorithms to be lower than that of the implementations of the randomization method developed in (Suñé and Carrasco, 2005) and lower than the run-time 
computational cost of the approach that consists in using iteratively the algorithms developed in (Sericola, 1999).

Not involving more subtractions than those required for the computation of the lower bounds (8), (27), and (29), which, as argued in Sections 2 and 3, should not result in significant numerical cancellations, and assuming that the involved Poisson probabilities are computed using a method with good numerical properties such as the one described in (Knüsel, 1986, pp. 1028-1029) (see also (Abramowitz and Stegun, 1964)), or, for sums of the form $\sum_{j=0}^{n} P_{j}(\Lambda t)$, the method described in Bowerman et al. (1990), the proposed algorithms are numerically stable.

For medium-sized and large MRMs, we can expect the run-time computational cost of the proposed algorithms to be dominated by the matrix-vector multiplies (MVMs) with matrix B. For Algorithm 1, the number of such MVMs will be equal to the value of the integer $K_{1}$ defined by (14) with $t$ replaced by $t_{n}$ and, for Algorithm 2, it will be equal to the value of the integer $K_{2}$ defined by (36) with $t$ replaced by $t_{n}$. However, in general it seems difficult to anticipate the values of $K_{1}$ or $K_{2}$.

The implementation of the randomization method developed in (Suñé and Carrasco, 2005) for the computation of $\operatorname{ETRR}(t)$, which will be referred to as Algorithm SC1, allows to compute $\operatorname{ETRR}(t)$ for a set of time points $t_{1}<\cdots<t_{n}$ and will involve a number of MVMs with matrix $\mathbf{B}$ equal to

$$
K_{1}^{\prime}=\min \left\{k \geq 0: \frac{1-\sum_{j=0}^{k} P_{j}\left(\Lambda t_{n}\right)}{\sum_{j=0}^{k} v_{j} P_{j}\left(\Lambda t_{n}\right)} \leq \frac{\varepsilon}{4}\right\} .
$$

The implementation of the randomization method developed in (Suñé and Carrasco, 2005) for the computation of $\operatorname{EARR}(t)$, which will be referred to as Algorithm SC2, allows to compute $\operatorname{EARR}(t)$ for a set of time points $t_{1}<\cdots<t_{n}$ and will involve a number of MVMs with matrix $\mathbf{B}$ equal to

$$
K_{2}^{\prime}=\min \left\{k \geq 0: \frac{1-\sum_{j=0}^{k} P_{j}\left(\Lambda t_{n}\right)}{\sum_{j=0}^{k} w_{j} P_{j}\left(\Lambda t_{n}\right)} \leq \frac{\varepsilon}{4}\right\} .
$$

For medium-sized and large MRMs, we can expect those MVMs to dominate the run-time computational cost of Algorithms SC1 and SC2. But, by (13), (14), using that, as assumed, $\varepsilon>\delta /(1-\delta)$, the parameter $K_{1}$ of Algorithm 1 will satisfy

$$
K_{1}=\min \left\{k \geq 0:\left(M_{k}-m_{k}\right) \frac{1-\sum_{j=0}^{k} P_{j}\left(\Lambda t_{n}\right)}{\sum_{j=0}^{k} v_{j} P_{j}\left(\Lambda t_{n}\right)} \leq \varepsilon_{1}\right\}
$$

with

$$
\varepsilon_{1}=\left(2+\left(m_{k}+M_{k}\right) \frac{1-\sum_{j=0}^{k} P_{j}\left(\Lambda t_{n}\right)}{\sum_{j=0}^{k} v_{j} P_{j}\left(\Lambda t_{n}\right)}(1-\delta(1+1 / \varepsilon))\right) \varepsilon>2 \varepsilon,
$$

and, by $(22),(35),(36)$, the parameter $K_{2}$ of Algorithm 2 will satisfy

$$
K_{2}=\min \left\{k \geq 0:\left(M_{k}-m_{k}\right) \frac{1-\sum_{j=0}^{k} P_{j}\left(\Lambda t_{n}\right)}{\sum_{j=0}^{k} w_{j} P_{j}\left(\Lambda t_{n}\right)} \leq \varepsilon_{2}\right\}
$$

with

$$
\begin{aligned}
\varepsilon_{2}= & \left(2+\left(m_{k}+M_{k}\right) \frac{\sum_{j=k+1}^{\infty} \frac{j-k}{j+1} P_{j}\left(\Lambda t_{n}\right)}{\sum_{j=0}^{k} w_{j} P_{j}\left(\Lambda t_{n}\right)}(1-\delta(1+1 / \varepsilon))+\frac{M_{k}-m_{k}}{\varepsilon} \frac{k+1}{\Lambda t}\right. \\
& \left.\times \frac{1-\sum_{j=0}^{k+1} P_{j}\left(\Lambda t_{n}\right)}{\sum_{j=0}^{k} w_{j} P_{j}\left(\Lambda t_{n}\right)}\right) \varepsilon>2 \varepsilon .
\end{aligned}
$$


Also, since $0 \leq 1-r_{\min } / r_{\max }=M_{0}-m_{0} \leq 1$ and the sequences $\left\{M_{k}\right\}$ and $\left\{m_{k}\right\}$ are, respectively, nonincreasing and nondecreasing (Sericola, 1999), it follows that $0 \leq M_{k}-m_{k} \leq 1, k \geq 0$. Therefore, we will always have $K_{1} \leq K_{1}^{\prime}$ and $K_{2} \leq K_{2}^{\prime}$. Further, for large enough $\Lambda t_{n}$, we will have $\sum_{j=0}^{K_{1}^{\prime}} v_{j} P_{j}\left(\Lambda t_{n}\right) \approx \operatorname{ETRR}(\infty)$ and $\sum_{j=0}^{K_{2}^{\prime}} w_{j} P_{j}\left(\Lambda t_{n}\right) \approx \operatorname{EARR}(\infty)$. Thus, for large enough $\Lambda t_{n}$, both (39) $K_{1}^{\prime}$ and (40) $K_{2}^{\prime}$ will depend, essentially, on the course of $1-\sum_{j=0}^{k} P_{j}\left(\Lambda t_{n}\right)$. But, for $\Lambda t_{n} \rightarrow \infty$, a Poisson distribution with parameter $\Lambda t_{n}$ has an asymptotic normal distribution with mean and variance $\Lambda t_{n}$. Then, for large enough $\Lambda t_{n}$ and $\varepsilon \ll 1$, the parameters $K_{1}^{\prime}$ and $K_{2}^{\prime}$ will be of the order of $\Lambda t_{n}$. On the other hand, as already argued at the end of Sections 2 and 3 , for large enough $\Lambda t_{n}$, the parameters $K_{1}$ and $K_{2}$ will be almost independent of $\Lambda t_{n}$. Therefore, for large enough $\Lambda t_{n}$, the differences $K_{1}^{\prime}-K_{1}$ and $K_{2}^{\prime}-K_{2}$ will increase with $\Lambda t_{n}$. In summary, for medium-sized and large MRMs, we can expect the run-time computational cost of Algorithm 1 to be lower than that of Algorithm SC1, can expect the run-time computational cost of Algorithm 2 to be lower than that of Algorithm SC2, and the larger $\Lambda t_{n}$, the larger we can expect to be the difference in run-time computational cost between Algorithms 1 and SC1 and between Algorithms 2 and SC2.

Let us now describe reasonable schemes to compute $\operatorname{ETRR}\left(t_{i}\right)$ and $\operatorname{EARR}\left(t_{i}\right), 1 \leq i \leq n$, with control of the relative error based on using iteratively the two algorithms developed in (Sericola, 1999). The first such algorithm computes $\operatorname{ETRR}\left(t_{i}\right), 1 \leq i \leq n$, with control of the absolute error and the second one computes $\operatorname{EARR}\left(t_{i}\right), 1 \leq i \leq n$, with control of the absolute error. Therefore, $\operatorname{ETRR}\left(t_{i}\right), 1 \leq i \leq n$, can be computed by invoking the first algorithm with an absolute error tolerance $\nu^{(1)}=\varepsilon\left(r_{\min }+r_{\max }\right) / 2$, say, and, next, if $\nu^{(1)} / \min _{1 \leq i \leq n} \widehat{\operatorname{ETRR}}^{(1)}\left(t_{i}\right)>\varepsilon$, where $\widehat{\operatorname{ETRR}}^{(j)}\left(t_{i}\right)$ denotes the approximation for $\operatorname{ETRR}\left(t_{i}\right)$ computed by the algorithm in the course of invocation $j$ with the convention $\widehat{\operatorname{ETRR}}^{(0)}\left(t_{i}\right)=\left(r_{\min }+r_{\max }\right) / 2$, continue invoking iteratively the algorithm with an absolute error tolerance

$$
\nu^{(j)}=\nu^{(j-1)} \min \left\{\frac{\min _{1 \leq i \leq n} \widehat{\operatorname{ETRR}}^{(j-1)}\left(t_{i}\right)}{\min _{1 \leq i \leq n} \widehat{\operatorname{ETRR}}^{(j-2)}\left(t_{i}\right)}, 0.95\right\}
$$

for invocation $j, j>1$, until $\nu^{(j)} / \min _{1 \leq i \leq n} \widehat{\operatorname{ETRR}}^{(j)}\left(t_{i}\right) \leq \varepsilon$. (The 0.95 is a security factor to help ensure convergence.) Similarly, in the iterative scheme for the computation of $\operatorname{EARR}\left(t_{i}\right), 1 \leq i \leq n$, we can start by invoking the second algorithm developed in (Sericola, 1999) with an absolute error tolerance $\nu^{(1)}=\varepsilon\left(r_{\min }+r_{\max }\right) / 2$ and, next, if $\nu^{(1)} / \min _{1 \leq i \leq n} \widehat{\operatorname{EARR}}^{(1)}\left(t_{i}\right)>\varepsilon$, where $\widehat{\operatorname{EARR}}^{(j)}\left(t_{i}\right)$ denotes the approximation for $\operatorname{EARR}\left(t_{i}\right)$ computed by the algorithm in the course of invocation $j$ with the convention $\widehat{\operatorname{EARR}}^{(0)}\left(t_{i}\right)=\left(r_{\min }+r_{\max }\right) / 2$, continue invoking iteratively the algorithm with an absolute error tolerance

$$
\nu^{(j)}=\nu^{(j-1)} \min \left\{\frac{\min _{1 \leq i \leq n} \widehat{\operatorname{EARR}}^{(j-1)}\left(t_{i}\right)}{\min _{1 \leq i \leq n} \widehat{\operatorname{EARR}}^{(j-2)}\left(t_{i}\right)}, 0.95\right\}
$$

for invocation $j, j>1$, until $\nu^{(j)} / \min _{1<i<n} \widehat{\operatorname{EARR}}^{(j)}\left(t_{i}\right) \leq \varepsilon$. For the sake of clarity, the iterative schemes for the computation of $\operatorname{ETRR}(t)$ and $\operatorname{EARR}(t)$ just described will be referred to as Algorithm SE1 and Algorithm SE2, respectively. Let $S_{1}$ denote the number of times that the first algorithm developed in (Sericola, 1999) is invoked in Algorithm SE1. Invocation $j, 1 \leq j \leq S_{1}$, of that algorithm will involve a number of MVMs with matrix $\mathbf{B}$ equal to $\min \left\{N^{(j)}, K^{(j)}\right\}$, where

$$
\begin{aligned}
& N^{(j)}=\min \left\{n \geq 0: r_{\max }\left(1-\sum_{j=0}^{n} P_{j}\left(\Lambda t_{n}\right)\right) \leq \nu^{(j)}\right\}, \\
& K^{(j)}=\min \left\{k \geq 0: r_{\max }\left(M_{k}-m_{k}\right) \leq \nu^{(j)} / 2\right\} .
\end{aligned}
$$


Therefore, Algorithm SE1 will involve $\sum_{i=1}^{S_{1}} \min \left\{N^{(i)}, K^{(i)}\right\}$ MVMs with matrix B and the integer $S_{1}$ will satisfy

$$
\frac{r_{\max } \min \left\{1-\sum_{j=0}^{N^{\left(S_{1}\right)}} P_{j}\left(\Lambda t_{n}\right), 2\left(M_{K^{\left(S_{1}\right)}}-m_{K^{\left(S_{1}\right)}}\right)\right\}}{\min _{1 \leq i \leq n} \widehat{\operatorname{ETRR}}^{\left(S_{1}\right)}\left(t_{i}\right)} \leq \varepsilon .
$$

For medium-sized and large MRMs, we can expect those MVMs to dominate the run-time computational cost of Algorithm SE1. However, by (11), (13), (14), assuming $\widehat{\operatorname{ETRR}}^{\left(S_{1}\right)}\left(t_{n}\right) \approx \widehat{\operatorname{ETRR}}\left(t_{n}, K_{1}\right)$, the parameter $K_{1}$ of Algorithm 1 will satisfy

$$
\begin{aligned}
\frac{r_{\max }\left(M_{K_{1}}-m_{K_{1}}\right)\left(1-\sum_{j=0}^{K_{1}} P_{j}\left(\Lambda t_{n}\right)\right)}{\widehat{\operatorname{ETRR}}^{\left(S_{1}\right)}\left(t_{n}\right)} & \approx \frac{r_{\max }\left(M_{K_{1}}-m_{K_{1}}\right)\left(1-\sum_{j=0}^{K_{1}} P_{j}\left(\Lambda t_{n}\right)\right)}{\widehat{\operatorname{ETRR}}\left(t_{n}, K_{1}\right)} \\
& \leq \frac{\left(M_{K_{1}}-m_{K_{1}}\right)\left(1-\sum_{j=0}^{K_{1}} P_{j}\left(\Lambda t_{n}\right)\right)}{\sum_{j=0}^{K_{1}} v_{j} P_{j}\left(\Lambda t_{n}\right)} \\
& \leq 2 \varepsilon+\left(m_{K_{1}}+M_{K_{1}}\right) \frac{1-\sum_{j=0}^{K_{1}} P_{j}\left(\Lambda t_{n}\right)}{\sum_{j=0}^{K_{1}} v_{j} P_{j}\left(\Lambda t_{n}\right)} \varepsilon^{\prime},
\end{aligned}
$$

which is $>2 \varepsilon$. Therefore, taking into account that

$$
\left.\left(M_{k}-m_{k}\right)\left(1-\sum_{j=0}^{k} P_{j}\left(\Lambda t_{n}\right)\right) \leq \min \left\{\left(1-\sum_{j=0}^{k} P_{j}\left(\Lambda t_{n}\right)\right), M_{k}-m_{k}\right)\right\}
$$

that the left-hand side of the above inequality is decreasing on $k$, and that $1 / \min _{1 \leq i \leq n} \widehat{\operatorname{ETRR}}^{\left(S_{1}\right)}\left(t_{i}\right) \geq$ $1 / \widehat{\operatorname{ETRR}}^{\left(S_{1}\right)}\left(t_{n}\right)$, we can expect $K_{1} \leq \min \left\{N^{\left(S_{1}\right)}, K^{\left(S_{1}\right)}\right\} \leq \sum_{i=1}^{S_{1}} \min \left\{N^{(i)}, K^{(i)}\right\}$. In addition, given the way Algorithm SE1 works, we can expect $S_{1}=1$ if $\min _{1 \leq i \leq n} \operatorname{ETRR}\left(t_{i}\right)$ is not smaller than $\left(r_{\min }+r_{\max }\right) / 2$ and otherwise can expect $S_{1} \geq 2$. Therefore, for medium-sized and large MRMs, we can expect the run-time computational cost of Algorithm 1 to be lower than that of Algorithm SE1, and the smaller $\min _{1 \leq i \leq n} \operatorname{ETRR}\left(t_{i}\right)$ than $\left(r_{\min }+r_{\max }\right) / 2$, the larger we can expect the difference in run-time computational cost to be.

Let $S_{2}$ denote the number of times the second algorithm developed in (Sericola, 1999) is invoked in Algorithm SE2. Invocation $j, 1 \leq j \leq S_{2}$, of that algorithm involves a number of MVMs with matrix B equal to $\min \left\{N^{(j)}, K^{(j)}\right\}$, where $N^{(j)}$ and $K^{(j)}$ are given by, respectively, (41) and (42). Therefore, Algorithm SE2 will involve $\sum_{j=1}^{S_{2}} \min \left\{N^{(j)}, K^{(j)}\right\}$ MVMs with matrix B and the integer $S_{2}$ will satisfy

$$
\frac{r_{\max } \min \left\{1-\sum_{j=0}^{N^{\left(S_{2}\right)}} P_{j}\left(\Lambda t_{n}\right), 2\left(M_{K^{\left(S_{2}\right)}}-m_{K^{\left(S_{2}\right)}}\right)\right\}}{\min _{1 \leq i \leq n} \widehat{\operatorname{EARR}}^{\left(S_{2}\right)}\left(t_{i}\right)} \leq \varepsilon .
$$

For medium-sized and large MRMs, we can expect those MVMs to dominate the run-time computational cost of Algorithm SE2. On the other hand, by (34), (35), (36), (22), assuming $\widehat{\operatorname{EARR}}^{\left(S_{2}\right)}\left(t_{n}\right) \approx \widehat{\operatorname{EARR}}\left(t_{i}, K_{2}\right)$, the parameter $K_{2}$ in Algorithm 2 will satisfy

$$
\begin{aligned}
\frac{r_{\max }\left(M_{K_{2}}-m_{K_{2}}\right)\left(P_{K_{2}+1}(\Lambda t)+\frac{\Lambda t-\left(K_{2}+1\right)}{\Lambda t}\left(1-\sum_{j=0}^{K_{2}+1} P_{j}\left(\Lambda t_{n}\right)\right)\right)}{\widehat{\operatorname{EARR}}^{\left(S_{2}\right)}\left(t_{n}\right)} \\
\approx \frac{r_{\max }\left(M_{K_{2}}-m_{K_{2}}\right)\left(P_{K_{2}+1}(\Lambda t)+\frac{\Lambda t-\left(K_{2}+1\right)}{\Lambda t}\left(1-\sum_{j=0}^{K_{2}+1} P_{j}\left(\Lambda t_{n}\right)\right)\right)}{\widehat{\operatorname{EARR}}\left(t_{n}, K_{2}\right)}
\end{aligned}
$$




$$
\begin{aligned}
& \leq \frac{r_{\max }\left(M_{K_{2}}-m_{K_{2}}\right)\left(P_{K_{2}+1}(\Lambda t)+\frac{\Lambda t-\left(K_{2}+1\right)}{\Lambda t}\left(1-\sum_{j=0}^{K_{2}+1} P_{j}\left(\Lambda t_{n}\right)\right)\right)}{r_{\max } \sum_{j=0}^{K_{2}} w_{j} P_{j}\left(\Lambda t_{n}\right)} \\
& \leq 2 \varepsilon+\frac{P_{K_{2}+1}(\Lambda t)+\frac{\Lambda t-\left(K_{2}+1\right)}{\Lambda t}\left(1-\sum_{j=0}^{K_{2}+1} P_{j}\left(\Lambda t_{n}\right)\right)}{\sum_{j=0}^{K_{2}} w_{j} P_{j}\left(\Lambda t_{n}\right)}\left(m_{K_{2}}+M_{K_{2}}\right) \varepsilon^{\prime} \\
& =2 \varepsilon+\frac{\sum_{j=K_{2}+1}^{\infty} \frac{j-K_{2}}{j+1} P_{j}(\Lambda t)}{\sum_{j=0}^{K_{2}} w_{j} P_{j}\left(\Lambda t_{n}\right)}\left(m_{K_{2}}+M_{K_{2}}\right) \varepsilon^{\prime} .
\end{aligned}
$$

which is $>2 \varepsilon$. Therefore, taking into account that, by (22),

$$
\begin{aligned}
\left(M_{k}-m_{k}\right)\left(P_{k+1}(\Lambda t)\right. & \left.+\frac{\Lambda t-(k+1)}{\Lambda t}\left(1-\sum_{j=0}^{k+1} P_{j}(\Lambda t)\right)\right) \\
& =\left(M_{k}-m_{k}\right)\left(1-\sum_{j=0}^{k} P_{j}\left(\Lambda t_{n}\right)-\frac{k+1}{\Lambda t_{n}}\left(1-\sum_{j=0}^{k+1} P_{j}\left(\Lambda t_{n}\right)\right)\right) \\
& \leq\left(M_{k}-m_{k}\right)\left(1-\sum_{j=0}^{k} P_{j}\left(\Lambda t_{n}\right)\right) \\
& \left.\leq \min \left\{\left(1-\sum_{j=0}^{k} P_{j}\left(\Lambda t_{n}\right)\right), M_{k}-m_{k}\right)\right\},
\end{aligned}
$$

that, again by (22), the left-hand side of the above inequality is decreasing on $k$, and that $1 / \min _{1 \leq i \leq n} \widehat{\operatorname{EARR}}^{\left(S_{2}\right)}\left(t_{i}\right) \geq 1 / \widehat{\operatorname{EARR}}^{\left(S_{2}\right)}\left(t_{n}\right)$, we can expect $K_{2} \leq \min \left\{N^{\left(S_{2}\right)}, K^{\left(S_{2}\right)}\right\} \leq$ $\sum_{j=1}^{S_{2}} \min \left\{N^{(j)}, K^{(j)}\right\}$. Further, given the way Algorithm SE2 works, we can expect $S_{2}=1$ if $\min _{1 \leq i \leq n} \operatorname{EARR}\left(t_{i}\right)$ is not smaller than $\left(r_{\text {min }}+r_{\max }\right) / 2$ and otherwise can expect $S_{2} \geq 2$. Therefore, we also conclude that, for medium-sized and large MRMs, we can expect the run-time computational

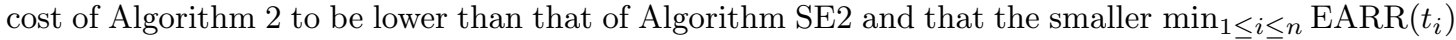
than $\left(r_{\min }+r_{\max }\right) / 2$, the larger we can expect the difference in run-time computational cost to be.

\section{Numerical Experiments}

In this section, we will illustrate the performances of Algorithms 1 and 2. We will also compare the performance of Algorithm 1 with the performances of Algorithms SC1 and SE1 and will compare the performance of Algorithm 2 with the performances of Algorithms SC2 and SE2.

\subsection{Examples}

We will use two MRMs. The first one is taken from (Carrasco, 2003b) and corresponds to a RAID 5 storage system (Chen et al., 1994) with the architecture shown in Figure 1. The system comprises $40 \times 5$ disks, 5 controllers, 3 hot spare disks, and 1 hot spare controller. The disks are organized into 40 parity groups with 5 disks each. Each controller controls a string of 40 disks. A disk is said to be unavailable if it has failed, or the controller of the string the disk belongs to has failed, or the data in the disk is out of date. The system is in a failed state if there is any parity group in which two or more disks are unavailable. The data of a non-failed disk becomes out of date if it is a disk that has just replaced a failed one or it belongs to a string of disks whose controller was failed and has just been replaced. Out-of-date disks become up-to-date after a reconstruction process that proceeds at a rate of $1 \mathrm{~h}^{-1}$. That process has a success probability 0.999 and can take place only 


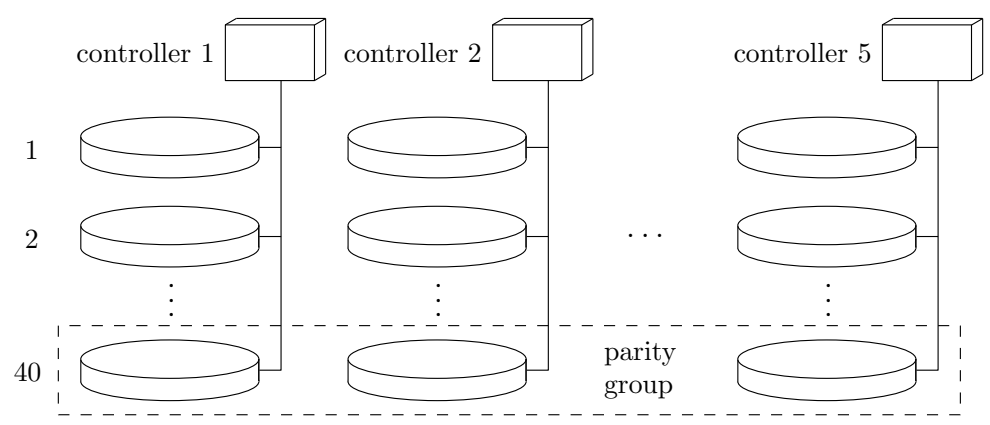

Fig. 1 Architecture of the RAID 5 storage system.

when the system is in a non-failed state. All disks of a parity group involved in a reconstruction process fail at a rate of $2 \times 10^{-5} \mathrm{~h}^{-1}$. Disks not involved in a reconstruction process fail at a rate of $1 \times 10^{-5} \mathrm{~h}^{-1}$. Controllers fail at a rate of $5 \times 10^{-5} \mathrm{~h}^{-1}$. There is one repair person that, if hot spares are available, replaces failed disks and controllers at a rate of $4 \mathrm{~h}^{-1}$, with priority given to controllers. There is an unlimited number of repair persons that replace used hot spares and failed disks and controllers when no hot spares are available at a rate of $0.25 \mathrm{~h}^{-1}$. When the system is in a failed state, no components fail or are repaired and the only repair action is one that brings the system to its fully operational state, with all disks in the parity groups available and all hot spares available, at a rate of $0.25 \mathrm{~h}^{-1}$. The initial probability is one for the fully operational state and is zero for the remaining states. As in (Carrasco, 2003b), we assume that if unavailable disks do not belong to the same string, when one of them becomes available the remaining unavailable disks still belong to different strings whenever their number is $\geq 2$. The CTMC has 14081 states and 94405 transition rates. The reward rates are $r_{i}=1$ for the nonfailed states and $r_{i}=0$ for the failed states. With those reward rates, $\operatorname{ETRR}(t)$ is the availability of the system at time $t$ (probability that the system is not failed at time $t$ ) and $\operatorname{EARR}(t)$ is the expected interval availability of the system in the time interval $[0, t]$ (expected fraction of the time interval in which the system is not failed). In addition, $r_{\min }=0$, and $r_{\max }=1$.

The second MRM, adapted from (Carrasco, 2015), corresponds to two FIFO queues working in tandem. Each queue has a buffer with capacity for $N=100$ tasks. Tasks arrive on the first queue with rate $\lambda=2 \mathrm{~h}^{-1}$. When a task of the first queue is served, it is delivered to the second queue unless it is full, in which case the task is blocked until there is room for it in the second queue. The service rates are $\mu_{\mathrm{F}}=2.2 \mathrm{~h}^{-1}$ for the first queue and $\mu_{\mathrm{S}}=2.5 \mathrm{~h}^{-1}$ for the second one. The initial probability is one for the state in which there are no tasks in the system and is zero for the remaining states. The CTMC has 10301 states and 30499 transition rates. Its state transition diagram is shown in Figure 2, where the states in which there are $i$ tasks in the first queue and $j$ tasks in the second one and no task is blocked are labeled " $i, j$ ", and the states in which there are $i$ tasks in the first queue, $N$ tasks in the second queue, and a task of the first queue is blocked are labeled " $i, N$ ". The reward rates are the number of tasks in the system. With those reward rates, $\operatorname{ETRR}(t)$ is the expected number of tasks in the system at time $t$ and $\operatorname{EARR}(t)$ is the expected average number of tasks in the system in the time interval $[0, t]$. Besides, $r_{\min }=0$, and $r_{\max }=200$.

\subsection{Results}

All algorithms were implemented using the C programming language, with all floating-point computations performed using the IEEE754-1985 (IEEE754) double format, and were compiled using the standard GNU compiler collection C-compiler (Stallman et al., 2012) with the 02 optimization option. The input parameter $\delta$ of the proposed algorithms was set to $10^{3} \times 2^{-52}$. In all cases, Poisson 


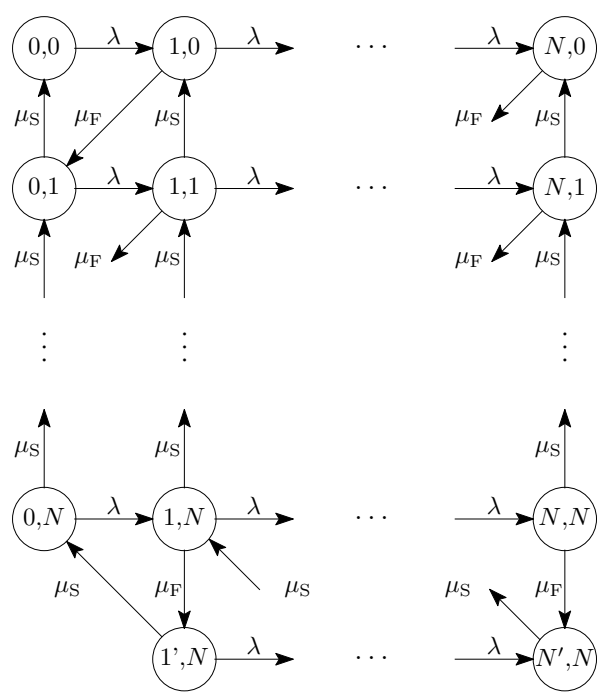

Fig. 2 State transition diagram of the queueing MRM.

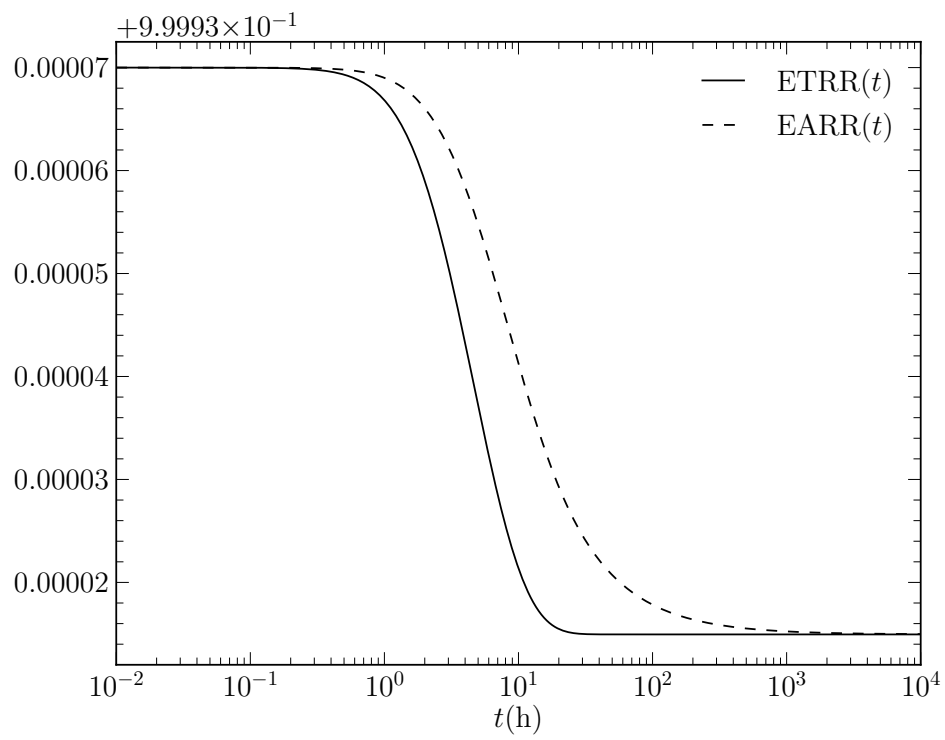

Fig. $3 \operatorname{ETRR}(t)$ and $\operatorname{EARR}(t)$ for the RAID 5 storage system MRM.

probabilities were computed using the method described in (Knüsel, 1986, pp. 1028-1029) (see also (Abramowitz and Stegun, 1964)) and $\Lambda$ was set to $\Lambda=\theta \times \max _{i \in \mathcal{S}}\left|a_{i, i}\right|$ with $\theta=1.001>1$ to ensure that $(2)$ holds. This latter setting resulted in $\Lambda \approx 43.80 \mathrm{~h}^{-1}$ for the RAID 5 storage system MRM and $\Lambda \approx 6.707 \mathrm{~h}^{-1}$ for the queueing MRM. All results were obtained on a workstation equipped with a four-core Intel i7-2630QM $2.00 \mathrm{GHz}$ processor with 4 GB of RAM memory, using only one core.

In Figures 3 and 4, we plot $\operatorname{ETRR}(t)$ and $\operatorname{EARR}(t)$ for the RAID 5 storage system MRM and the queueing MRM, respectively, obtained executing the proposed algorithms for 300 time points equally spaced in a logarithmic scale with a relative error tolerance $\varepsilon=10^{-10}$. We can observe that, for the first example, $\operatorname{ETRR}(t)$ "reaches" the stationary value very soon, at about $t=100 \mathrm{~h}$, that 


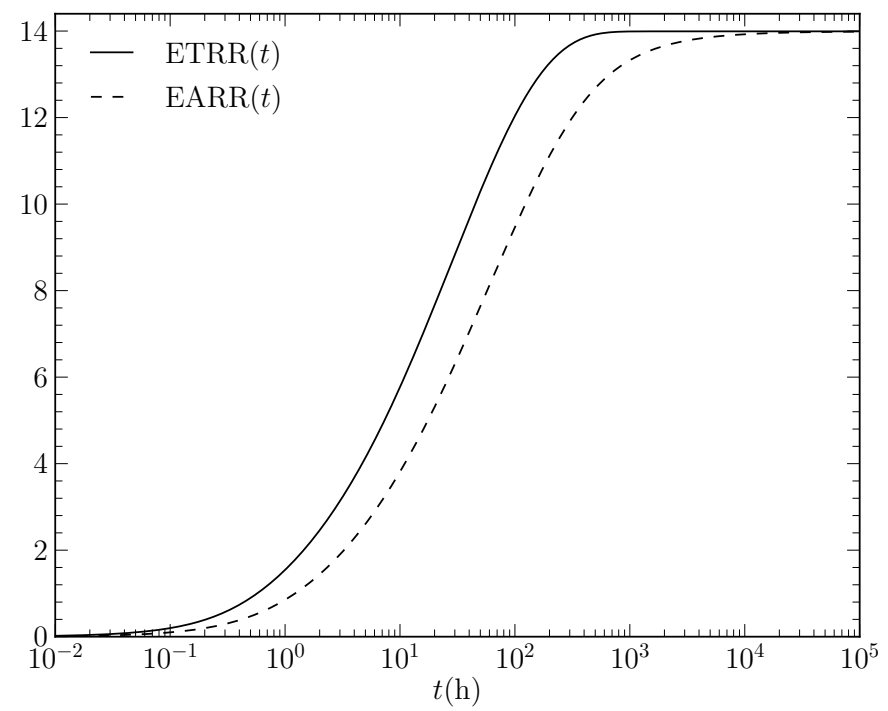

Fig. $4 \operatorname{ETRR}(t)$ and $\operatorname{EARR}(t)$ for the queueing MRM.

$\operatorname{EARR}(t)$ "reaches" the stationary value somewhat later, and that both $\operatorname{ETRR}(t)$ and $\operatorname{EARR}(t)$ are larger than $\left(r_{\min }+r_{\max }\right) / 2=0.5$. For the queueing MRM, we observe, both $\operatorname{ETRR}(t)$ and $\operatorname{EARR}(t)$ "reach" the stationary value later, between $t=1000 \mathrm{~h}$ and $t=10000 \mathrm{~h}$, and are significantly smaller than $\left(r_{\min }+r_{\max }\right) / 2=100$.

We will compare the proposed algorithms with the alternatives from a triple perspective: run-time computational cost measured in terms of CPU time, relative accuracy, and run-time computational cost in relation to relative accuracy. To carry out those comparisons, we executed each algorithm for both MRMs with $n=1, t_{n}=5 \mathrm{~h}, 10 \mathrm{~h}, \ldots, 10^{5} \mathrm{~h}$, and $\varepsilon=10^{-4}, 10^{-5}, \ldots, 10^{-12}$. The reference values for $\operatorname{ETRR}(t)$ and $\operatorname{EARR}(t), t=5 \mathrm{~h}, 10 \mathrm{~h}, \ldots, 10^{5} \mathrm{~h}$, with which to compute the algorithms' accuracy were obtained using the implementation of the randomization method described in (Suñé and Carrasco, 2005, Section 1), computing Poisson probabilities using a variant of the algorithm described in (Fox and Glynn, 1988), which is numerically very stable, and performing all floatingpoint computations using the IEEE 754-2008 binary128 format (IEEE754-2008) emulated with the MPFR library (Fousse et al., 2007). (The binary128 format gives around 34 decimal digits precision as opposed to the approximately 16 decimal digits precision given by the double format.)

The numbers of MVMs with matrix $\mathbf{B}$ required by the proposed algorithms were always smaller than the numbers of MVMs with matrix $\mathbf{B}$ required by the alternatives and, consequently, the CPU times of the proposed algorithms were almost always lower than those of the alternatives. In addition, the larger $t_{n}$, the larger were the differences in terms of CPU time between Algorithm 1 and Algorithm SC1 and between Algorithm 2 and Algorithm SC2. As an illustration, in Tables 1, 2, 3, 4 we give the number of MVMs with matrix B and the CPU time for each of the algorithms, for $\varepsilon=10^{-6}, 10^{-10}$. The tables also illustrate the fact that, as commented in Section 4 , how the runtime computational cost of Algorithm 1 compares with that of Algorithm SE1 depends on whether $\min _{1 \leq i \leq n} \operatorname{ETRR}\left(t_{i}\right) \geq\left(r_{\min }+r_{\max }\right) / 2$, and how the run-time computational cost of Algorithm 2 compares with that of Algorithm SE2 depends on whether $\min _{1 \leq i \leq n} \operatorname{EARR}\left(t_{i}\right) \geq\left(r_{\min }+r_{\max }\right) / 2$. Thus, for the first MRM, for which both $\operatorname{ETRR}\left(t_{n}\right)$ and $\operatorname{EARR}\left(t_{n}\right), t_{n}=5 \mathrm{~h}, 10 \mathrm{~h}, \ldots, 10^{5} \mathrm{~h}$, are larger than $\left(r_{\min }+r_{\max }\right) / 2=0.5$, Algorithms SE1 and SE2 always terminated in one iteration and, as a consequence, Algorithm 1 was only slightly faster than Algorithm SE1 and Algorithm 2 was 


\begin{tabular}{|r|r|r|r||r|r|r|}
\hline \multirow{2}{*}{$t_{n}(\mathrm{~h})$} & \multicolumn{4}{|c|}{$\varepsilon=10^{-6}$} & \multicolumn{3}{|c|}{$\varepsilon=10^{-10}$} \\
\cline { 2 - 7 } & \multicolumn{1}{|c|}{ Alg. 1 } & \multicolumn{1}{|c|}{ Alg. SC1 } & \multicolumn{1}{|c|}{ Alg. SE1 } & \multicolumn{1}{c|}{ Alg. 1 } & \multicolumn{1}{|c|}{ Alg. SC1 } & Alg. SE1 \\
\hline \multirow{2}{*}{5} & $285(359)$ & 297 & 295 & $313(377)$ & 323 & 321 \\
& $4.80 \times 10^{-2}$ & $5.20 \times 10^{-2}$ & $4.80 \times 10^{-2}$ & $5.20 \times 10^{-2}$ & $5.60 \times 10^{-2}$ & $5.20 \times 10^{-2}$ \\
\hline \multirow{2}{*}{10} & $523(628)$ & 547 & 544 & $563(651)$ & 582 & 580 \\
& $8.40 \times 10^{-2}$ & $9.20 \times 10^{-2}$ & $8.80 \times 10^{-2}$ & $8.80 \times 10^{-2}$ & $1.00 \times 10^{-1}$ & $9.60 \times 10^{-2}$ \\
\hline \multirow{2}{*}{100} & $2293(\mathrm{NC})$ & 4712 & 2656 & $3902(\mathrm{NC})$ & 4817 & 4265 \\
& $3.72 \times 10^{-1}$ & $8.12 \times 10^{-1}$ & $4.32 \times 10^{-1}$ & $6.36 \times 10^{-1}$ & $8.28 \times 10^{-1}$ & $6.92 \times 10^{-1}$ \\
\hline \multirow{2}{*}{1000} & $2293(\mathrm{NC})$ & 44810 & 2656 & $3902(\mathrm{NC})$ & 45136 & 4265 \\
& $3.68 \times 10^{-1}$ & 7.74 & $4.32 \times 10^{-1}$ & $6.36 \times 10^{-1}$ & 7.77 & $6.96 \times 10^{-1}$ \\
\hline \multirow{2}{*}{10000} & $2293(\mathrm{NC})$ & 440871 & 2656 & $3902(\mathrm{NC})$ & 441895 & 4265 \\
& $3.72 \times 10^{-1}$ & $7.55 \times 10^{1}$ & $4.44 \times 10^{-1}$ & $6.32 \times 10^{-1}$ & $7.63 \times 10^{1}$ & $7.04 \times 10^{-1}$ \\
\hline \multirow{2}{*}{100000} & $2293(\mathrm{NC})$ & 4385937 & 2656 & $3902(\mathrm{NC})$ & 4389171 & 4265 \\
& $3.76 \times 10^{-1}$ & $7.57 \times 10^{2}$ & $5.24 \times 10^{-1}$ & $6.28 \times 10^{-1}$ & $7.52 \times 10^{2}$ & $7.88 \times 10^{-1}$ \\
\hline
\end{tabular}

Table 1 RAID 5 storage system MRM: numbers of MVMs with matrix B (top) and CPU times in seconds (bottom) required by Algorithms 1, SC1, and SE1 to compute $\operatorname{ETRR}\left(t_{n}\right), n=1$. (For Algorithm 1, next to the number of MVMs we give between parenthesis the value of the truncation parameter $R_{1}$, with "NC" standing for "not computed".)

\begin{tabular}{|r|r|r|r||r|r|r|}
\hline \multirow{2}{*}{$t_{n}(\mathrm{~h})$} & \multicolumn{4}{|c|}{$\varepsilon=10^{-6}$} & \multicolumn{4}{|c|}{$\varepsilon=10^{-10}$} \\
\cline { 2 - 7 } & \multicolumn{1}{|c|}{ Alg. 2} & \multicolumn{1}{|c|}{ Alg. SC2 } & Alg. SE2 & \multicolumn{1}{c|}{ Alg. 2 } & \multicolumn{1}{c|}{ Alg. SC2 } & Alg. SE2 \\
\hline \hline \multirow{2}{*}{5} & $269(352,357)$ & 297 & 295 & $300(369,374)$ & 323 & 321 \\
& $4.40 \times 10^{-2}$ & $5.20 \times 10^{-2}$ & $4.80 \times 10^{-2}$ & $5.20 \times 10^{-2}$ & $5.60 \times 10^{-2}$ & $5.20 \times 10^{-2}$ \\
\hline \multirow{2}{*}{10} & $498(617,624)$ & 547 & 544 & $544(640,647)$ & 582 & 580 \\
& $8.00 \times 10^{-2}$ & $9.20 \times 10^{-2}$ & $8.80 \times 10^{-2}$ & $8.80 \times 10^{-2}$ & $1.00 \times 10^{-1}$ & $9.60 \times 10^{-2}$ \\
\hline \multirow{2}{*}{100} & $2293(\mathrm{NC}, \mathrm{NC})$ & 4712 & 2656 & $3902(\mathrm{NC}, \mathrm{NC})$ & 4817 & 4265 \\
& $3.72 \times 10^{-1}$ & $8.08 \times 10^{-1}$ & $4.32 \times 10^{-1}$ & $6.36 \times 10^{-1}$ & $8.32 \times 10^{-1}$ & $6.92 \times 10^{-1}$ \\
\hline \multirow{2}{*}{1000} & $2293(\mathrm{NC}, \mathrm{NC})$ & 44810 & 2656 & $3902(\mathrm{NC}, \mathrm{NC})$ & 45136 & 4265 \\
& $3.68 \times 10^{-1}$ & 7.80 & $4.36 \times 10^{-1}$ & $6.36 \times 10^{-1}$ & 7.71 & $6.96 \times 10^{-1}$ \\
\hline \multirow{2}{*}{10000} & $2293(\mathrm{NC}, \mathrm{NC})$ & 440871 & 2656 & $3902(\mathrm{NC}, \mathrm{NC})$ & 441895 & 4265 \\
& $3.72 \times 10^{-1}$ & $7.66 \times 10^{1}$ & $4.44 \times 10^{-1}$ & $6.32 \times 10^{-1}$ & $7.60 \times 10^{1}$ & $7.04 \times 10^{-1}$ \\
\hline \multirow{2}{*}{100000} & $2293(\mathrm{NC}, \mathrm{NC})$ & 4385937 & 2656 & $3902(\mathrm{NC}, \mathrm{NC})$ & 4389171 & 4265 \\
& $3.72 \times 10^{-1}$ & $7.56 \times 10^{2}$ & $5.24 \times 10^{-1}$ & $6.28 \times 10^{-1}$ & $7.58 \times 10^{2}$ & $7.88 \times 10^{-1}$ \\
\hline
\end{tabular}

Table 2 RAID 5 storage system MRM: numbers of MVMs with matrix B (top) and CPU times in seconds (bottom) required by Algorithms 2, SC2, and SE2 to compute EARR $\left(t_{n}\right), n=1$. (For Algorithm 2, next to the number of MVMs we give between parenthesis the value of the truncation parameters $R_{2}$ and $R_{3}$, with "NC" standing for "not computed".)

\begin{tabular}{|r|r|r|r||r|r|r|}
\hline \multirow{2}{*}{$t_{n}(\mathrm{~h})$} & \multicolumn{4}{|c|}{$\varepsilon=10^{-6}$} & \multicolumn{4}{|c|}{$\varepsilon=10^{-10}$} \\
\cline { 2 - 7 } & \multicolumn{1}{|c|}{ Alg. 1 } & Alg. SC1 & Alg. SE1 & Alg. 1 & Alg. SC1 & \multicolumn{1}{c|}{ Alg. SE1 } \\
\hline \multirow{2}{*}{5} & $69(100)$ & 72 & 135 & $80(108)$ & 82 & 158 \\
& $4.00 \times 10^{-3}$ & $4.00 \times 10^{-3}$ & $8.00 \times 10^{-3}$ & $8.00 \times 10^{-3}$ & $4.00 \times 10^{-3}$ & $8.00 \times 10^{-3}$ \\
\hline \multirow{2}{*}{10} & $115(156)$ & 118 & 227 & $130(167)$ & 133 & 257 \\
& $8.00 \times 10^{-3}$ & $8.00 \times 10^{-3}$ & $1.60 \times 10^{-2}$ & $8.00 \times 10^{-3}$ & $1.20 \times 10^{-2}$ & $2.00 \times 10^{-2}$ \\
\hline \multirow{2}{*}{100} & $806(920)$ & 818 & 1613 & $848(947)$ & 858 & 1699 \\
& $5.60 \times 10^{-2}$ & $5.60 \times 10^{-2}$ & $1.12 \times 10^{-1}$ & $6.00 \times 10^{-2}$ & $6.00 \times 10^{-2}$ & $1.20 \times 10^{-1}$ \\
\hline \multirow{2}{*}{1000} & $7009(7404)$ & 7156 & 14253 & $7166(7490)$ & 7277 & 14511 \\
& $4.92 \times 10^{-1}$ & $5.00 \times 10^{-1}$ & 1.01 & $5.08 \times 10^{-1}$ & $5.04 \times 10^{-1}$ & 1.02 \\
\hline \multirow{2}{*}{10000} & $15912(\mathrm{NC})$ & 68432 & 49949 & $25001(\mathrm{NC})$ & 68808 & 77212 \\
& 1.12 & 4.78 & 3.52 & 1.77 & 4.81 & 5.43 \\
\hline \multirow{2}{*}{100000} & $15912(\mathrm{NC})$ & 674519 & 49949 & $25001(\mathrm{NC})$ & 675702 & 77212 \\
& 1.13 & $4.66 \times 10^{1}$ & 3.57 & 1.76 & $4.72 \times 10^{1}$ & 5.50 \\
\hline
\end{tabular}

Table 3 Queueing MRM: numbers of MVMs with matrix B (top) and CPU times in seconds (bottom) required by Algorithms 1, SC1, and SE1 to compute $\operatorname{ETRR}\left(t_{n}\right), n=1$. (For Algorithm 1, next to the number of MVMs we give between parenthesis the value of the truncation parameter $R_{1}$, with "NC" standing for "not computed".) 


\begin{tabular}{|c|c|c|c|c|c|c|}
\hline \multirow[b]{2}{*}{$t_{n}(\mathrm{~h})$} & \multicolumn{3}{|c|}{$\varepsilon=10^{-6}$} & \multicolumn{3}{|c|}{$\varepsilon=10^{-10}$} \\
\hline & Alg. 2 & Alg. SC2 & Alg. SE2 & Alg. 2 & Alg. SC2 & Alg. SE2 \\
\hline \multirow{2}{*}{5} & $64(98,99)$ & 72 & 135 & $76(106,108)$ & 83 & 158 \\
\hline & $4.00 \times 10^{-3}$ & $4.00 \times 10^{-3}$ & $1.20 \times 10^{-2}$ & $4.00 \times 10^{-3}$ & $4.00 \times 10^{-3}$ & $1.20 \times 10^{-2}$ \\
\hline \multirow{2}{*}{10} & $108(152,155)$ & 119 & 228 & $124(163,165)$ & 133 & 257 \\
\hline & $8.00 \times 10^{-3}$ & $8.00 \times 10^{-3}$ & $1.60 \times 10^{-2}$ & $1.20 \times 10^{-2}$ & $8.00 \times 10^{-3}$ & $2.00 \times 10^{-2}$ \\
\hline \multirow{2}{*}{100} & $780(906,915)$ & 819 & 1614 & $827(934,942)$ & 859 & 1700 \\
\hline & $5.60 \times 10^{-2}$ & $6.00 \times 10^{-2}$ & $1.12 \times 10^{-1}$ & $6.00 \times 10^{-2}$ & $6.00 \times 10^{-2}$ & $1.20 \times 10^{-1}$ \\
\hline \multirow{2}{*}{1000} & $6872(7351,7379)$ & 7157 & 14254 & $7072(7437,7465)$ & 7278 & 14511 \\
\hline & $4.88 \times 10^{-1}$ & $5.00 \times 10^{-1}$ & 1.01 & $4.96 \times 10^{-1}$ & $5.04 \times 10^{-1}$ & 1.02 \\
\hline \multirow{2}{*}{10000} & $15912(\mathrm{NC}, \mathrm{NC})$ & 68432 & 49958 & $25001(\mathrm{NC}, \mathrm{NC})$ & 68809 & 77221 \\
\hline & 1.12 & 4.73 & 3.55 & 1.76 & 4.77 & 5.48 \\
\hline \multirow{2}{*}{100000} & $15912(\mathrm{NC}, \mathrm{NC})$ & 674519 & 49950 & $25001(\mathrm{NC}, \mathrm{NC})$ & 675702 & 77212 \\
\hline & 1.12 & $4.65 \times 10^{1}$ & 3.56 & 1.77 & $4.70 \times 10^{1}$ & 5.52 \\
\hline
\end{tabular}

Table 4 Queueing MRM: numbers of MVMs with matrix B (top) and CPU times in seconds (bottom) required by Algorithms 2, SC2, and SE2 to compute $\operatorname{EARR}\left(t_{n}\right), n=1$. (For Algorithm 2, next to the number of MVMs we give between parenthesis the value of the truncation parameters $R_{2}$ and $R_{3}$, with "NC" standing for "not computed".)

only slightly faster than Algorithm SE2. On the contrary, for the second MRM, for which both $\operatorname{ETRR}\left(t_{n}\right)$ and $\operatorname{EARR}\left(t_{n}\right), t_{n}=5 \mathrm{~h}, 10 \mathrm{~h}, \ldots, 10^{5} \mathrm{~h}$, are much smaller than $\left(r_{\text {min }}+r_{\text {max }}\right) / 2=100$, Algorithms SE1 and SE2 always required between two and three iterations and were therefore noticeably slower than, respectively, Algorithms 1 and 2. Also given in the tables are the values taken by the truncation parameters $R_{1}, R_{2}$, and $R_{3}$. As we can see, for the RAID 5 storage system MRM, the parameters were computed only for $t_{n}=5,10 \mathrm{~h}$ and their values are larger than the numbers of MVMs with matrix B required by the alternatives, and for the queueing MRM, the parameters were computed for $t_{n}=5,10,100,1000 \mathrm{~h}$ and their values are moderately larger than the numbers of MVMs with matrix B required by Algorithms SC1 and SC2 and smaller than the numbers of MVMs with matrix $\mathbf{B}$ required by Algorithms SE1 and SE2. However, computing the truncation parameter (8) $R_{1}$ essentially amounts to obtaining $R_{1}+1-K_{1}$ additional Poisson probabilities and computing the truncation parameters (28) $R_{2}$ and (30) $R_{3}$ essentially amounts to obtaining $\max \left\{R_{2}, R_{3}+1\right\}-K_{2}$ additional Poisson probabilities. Therefore, for medium-sized and large MRMs, assuming, quite reasonably, that the run-time computational cost of performing one MVM with matrix $\mathbf{B}$ will be substantially higher than the run-time computational cost of computing one Poisson probability, we expect the computation of those parameters to have a very small impact on the run-time computational cost of the proposed algorithms. That this is certainly the case for the two MRMs we are considering can be easily realized by comparing the CPU times required by the proposed algorithms with those required by Algorithms SC1 and SC2 in the cases in which the truncation parameters were computed.

Since the proposed algorithms and the alternatives all control the approximation error relative to the computed estimate, we cannot expect Algorithms 1, SC1, and SE1 to yield the same approximation for $\operatorname{ETRR}(t)$ within the relative error tolerance nor can expect Algorithms 2, SC2, and SE2 to yield the same approximation for $\operatorname{EARR}(t)$ within the relative error tolerance. Instead, what must happen is that the error of each estimate relative to the estimate itself is nonlarger than $\varepsilon$. To compare, in terms of relative accuracy, Algorithm 1 with Algorithms SC1 and SE1, and Algorithm 2 with Algorithms SC2 and SE2, we computed the maximum over $t_{n}=5 \mathrm{~h}, 10 \mathrm{~h}, \ldots, 10^{5} \mathrm{~h}$ of the actual error relative to the computed estimate for each algorithm and each $\varepsilon=10^{-4}, 10^{-5}, \ldots, 10^{-12}$. The results are shown in Figures 5, 6 for the RAID 5 storage system MRM and in Figures 7, 8 for the queueing MRM. As we can see, the control of the error in the proposed algorithms is very tight in the sense that the actual relative error is always very close to (but smaller than) the tolerance $\varepsilon$. We also see that, in almost all cases, the proposed algorithms are less accurate than the alternatives. Finally, we note that Algorithms SC1 and SC2 were unable to fulfill the accuracy requirement for 


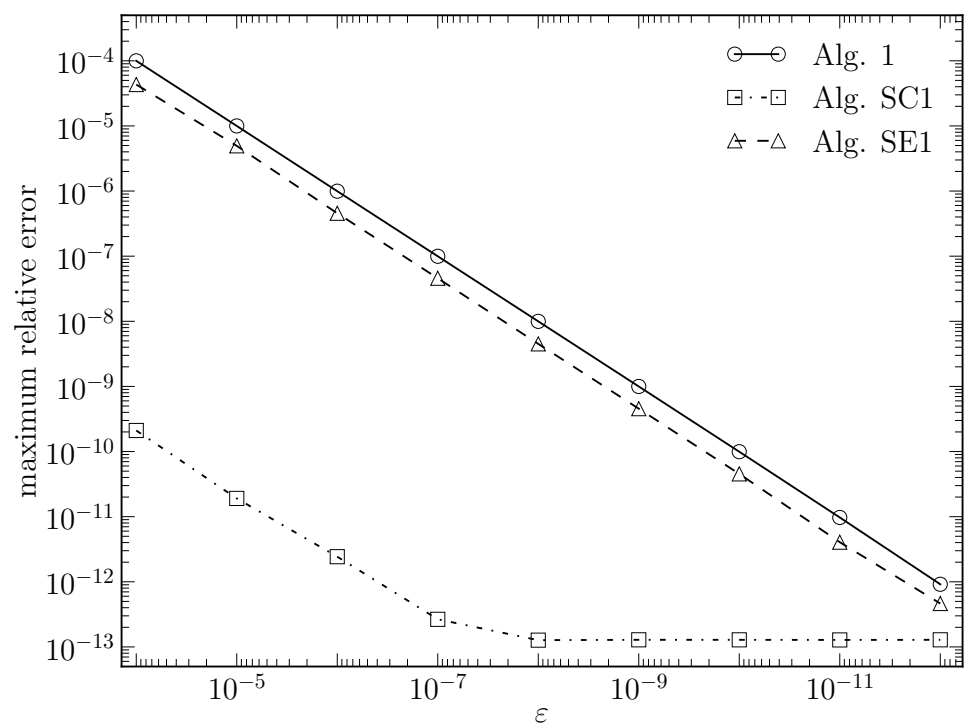

Fig. 5 RAID 5 storage system MRM: maximum over $t_{n}=5 \mathrm{~h}, 10 \mathrm{~h}, \ldots, 10^{5} \mathrm{~h}$ of the actual relative error in Algorithms 1, SC1, and SE1, as a function of $\varepsilon$.

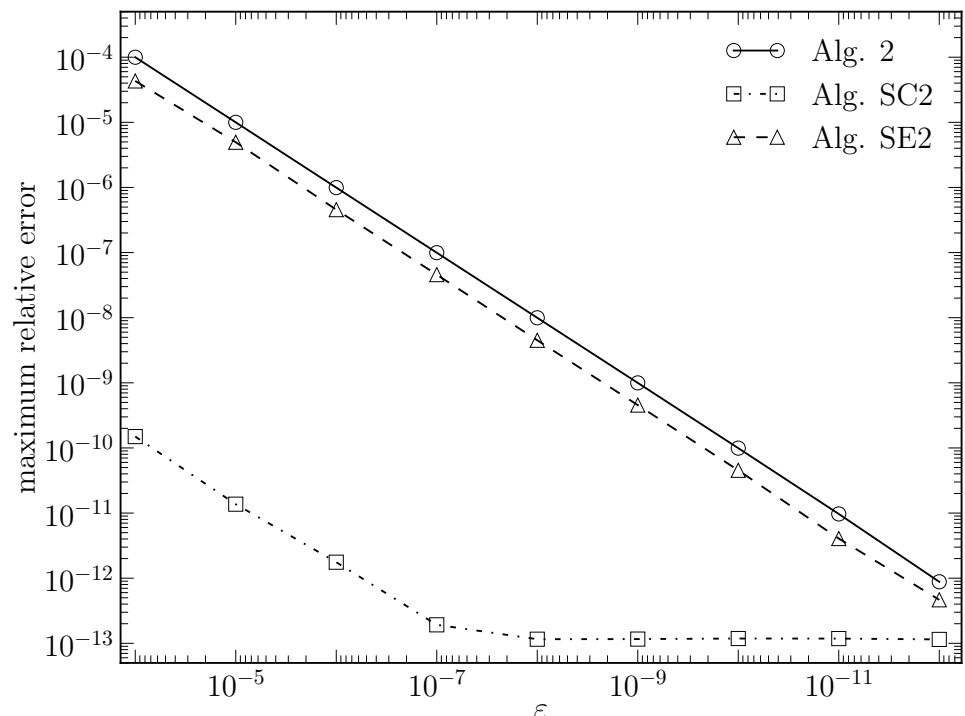

Fig. 6 RAID 5 storage system MRM: maximum over $t_{n}=5 \mathrm{~h}, 10 \mathrm{~h}, \ldots, 10^{5} \mathrm{~h}$ of the actual relative error in Algorithms 2, SC2, and SE2, as a function of $\varepsilon$. 


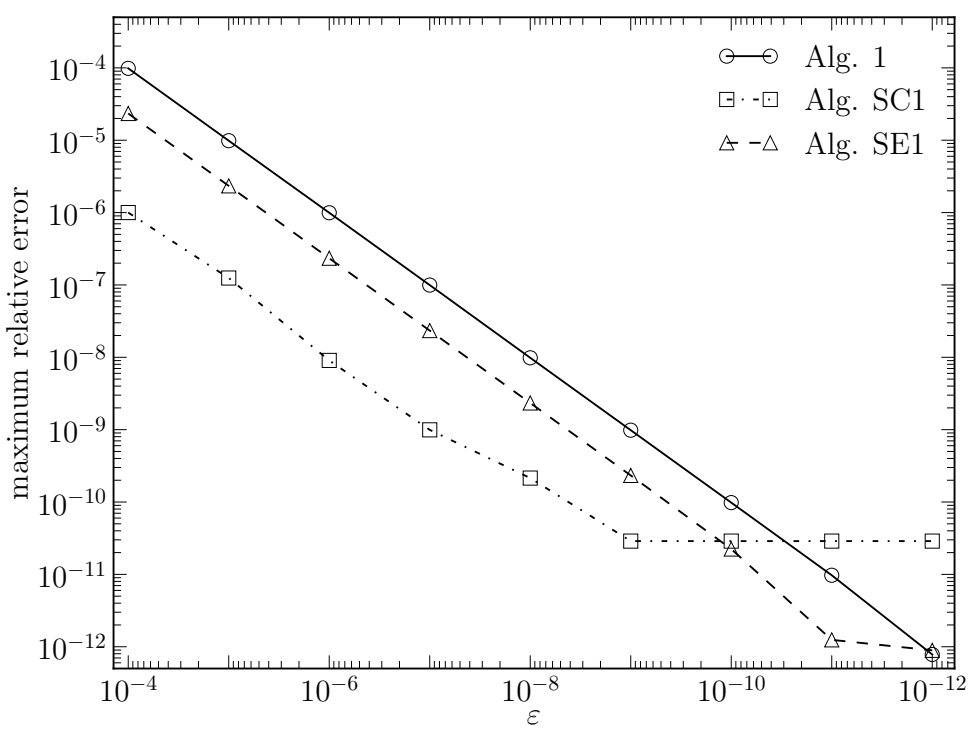

Fig. 7 Queueing MRM: maximum over $t_{n}=5 \mathrm{~h}, 10 \mathrm{~h}, \ldots, 10^{5} \mathrm{~h}$ of the actual relative error in Algorithms 1, SC1, and $\mathrm{SE} 1$, as a function of $\varepsilon$.

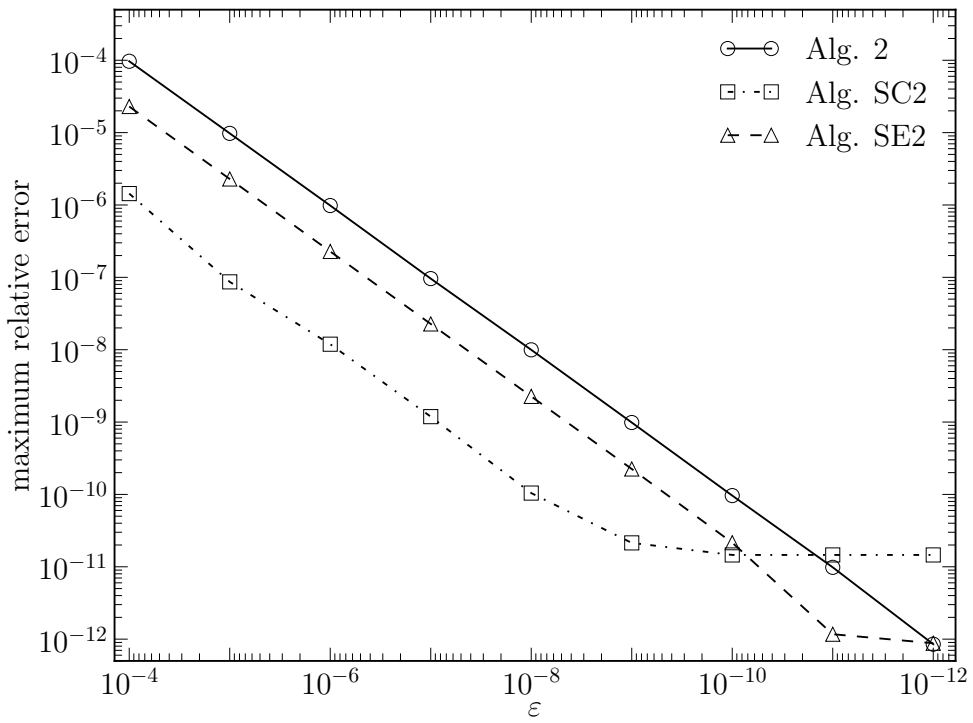

Fig. 8 Queueing MRM: maximum over $t_{n}=5 \mathrm{~h}, 10 \mathrm{~h}, \ldots, 10^{5} \mathrm{~h}$ of the actual relative error in Algorithms 2, $\mathrm{SC} 2$, and $\mathrm{SE} 2$, as a function of $\varepsilon$. 


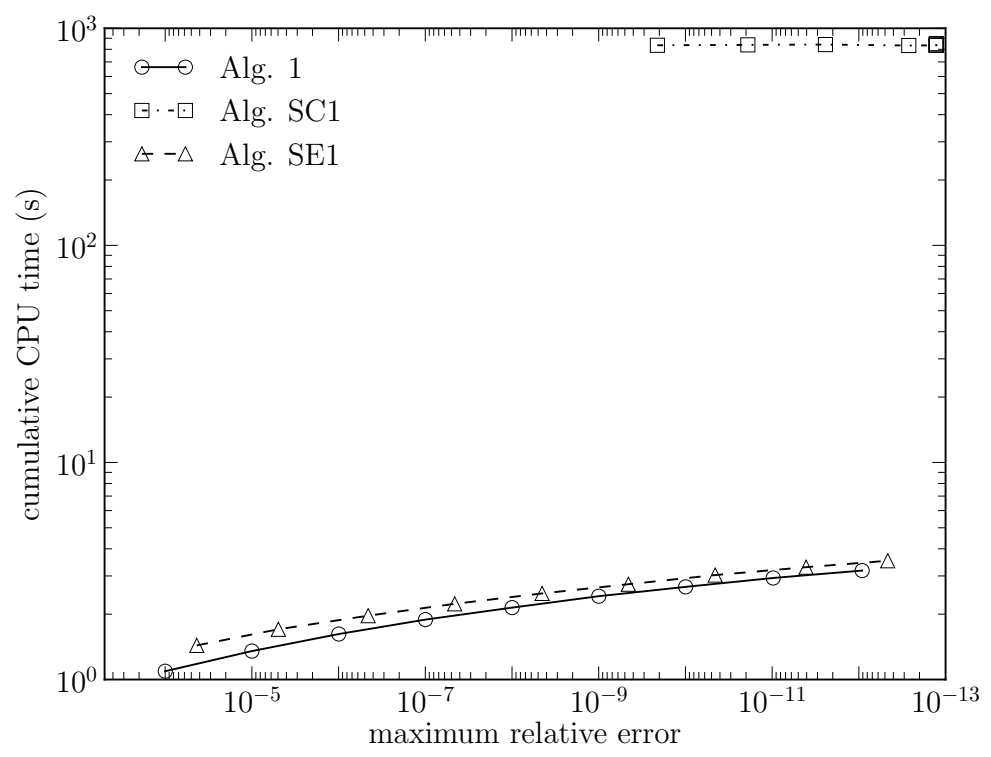

Fig. 9 RAID 5 storage system MRM: cumulative CPU time in seconds required to compute $\operatorname{ETRR}\left(t_{n}\right), t_{n}=$ $5 \mathrm{~h}, 10 \mathrm{~h}, \ldots, 10^{5} \mathrm{~h}$, as a function of the maximum over $t_{n}$ of the actual relative error in Algorithms 1 , SC1, and SE1. (In each case, the left-most symbol corresponds to $\varepsilon=10^{-4}$.)

the queueing MRM with $\varepsilon=10^{-11}, 10^{-12}$. We conjecture that this anomalous behavior is due to the cumulative effect of round-off errors.

The fact that the alternatives are more accurate than the proposed algorithms comes at the price of a higher run-time computational cost. Therefore, it would be fairer to compare the proposed algorithms with the alternatives from the perspective of relative accuracy in relation to run-time computational cost. To that end, in Figures 9, 10, 11, 12 we show the work-precision curves of the six algorithms. In each of these curves, the abscissa of the $i$ th point, $i=1,2, \ldots, 9$, starting from the left, corresponds to the maximum over $t_{n}=5 \mathrm{~h}, 10 \mathrm{~h}, \ldots, 10^{5} \mathrm{~h}$ of the actual relative error when the algorithm was executed with a relative error tolerance $\varepsilon=10^{-(3+i)}$, and the ordinate corresponds to the cumulative CPU time required by the algorithm for $t_{n}=5 \mathrm{~h}, 10 \mathrm{~h}, \ldots, 10^{5} \mathrm{~h}$. We can now see that, in all cases, Algorithms 1 and 2 are much more efficient than Algorithms SC1 and $\mathrm{SC} 2$, respectively, in the sense of requiring a much smaller CPU time to achieve the same relative error, that Algorithm 1 is slightly more efficient than Algorithm SE1 for the RAID 5 storage system MRM and quite more efficient for the queueing MRM, and that, compared with Algorithm SE2, Algorithm 2 is slightly more efficient for the RAID 5 storage system MRM and quite more so for the queueing MRM.

\section{Conclusions}

In this paper, by combining in a novel way the randomization method with the stationarity detection technique proposed in (Sericola, 1999), we have developed two new algorithms for the computation of the expected reward rates of finite, irreducible MRMs, with control of the relative error. The first algorithm computes the expected transient reward rate and the second one computes the expected averaged reward rate. We have argued that the algorithms are numerically stable and that, for medium-sized and large MRMs, we can expect the run-time computational cost of the 


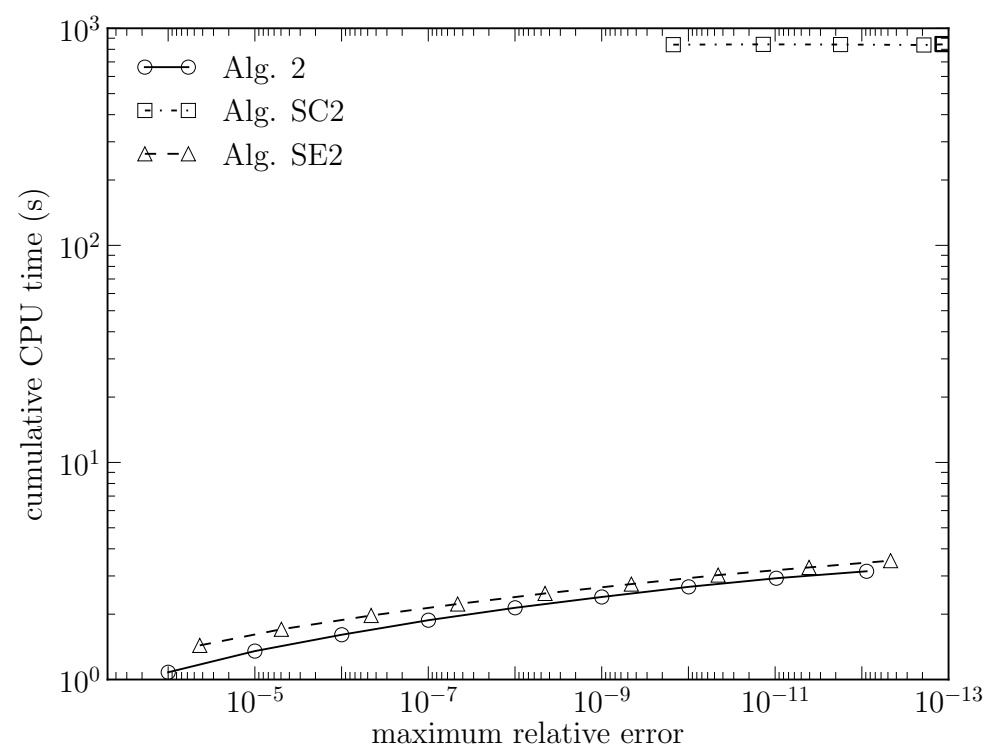

Fig. 10 RAID 5 storage system MRM: cumulative CPU time in seconds required to compute $\operatorname{EARR}\left(t_{n}\right)$, $t_{n}=5 \mathrm{~h}, 10 \mathrm{~h}, \ldots, 10^{5} \mathrm{~h}$, as a function of the maximum over $t_{n}$ of the actual relative error in Algorithms $2, \mathrm{SC} 2$, and SE2. (In each case, the left-most symbol corresponds to $\varepsilon=10^{-4}$.)

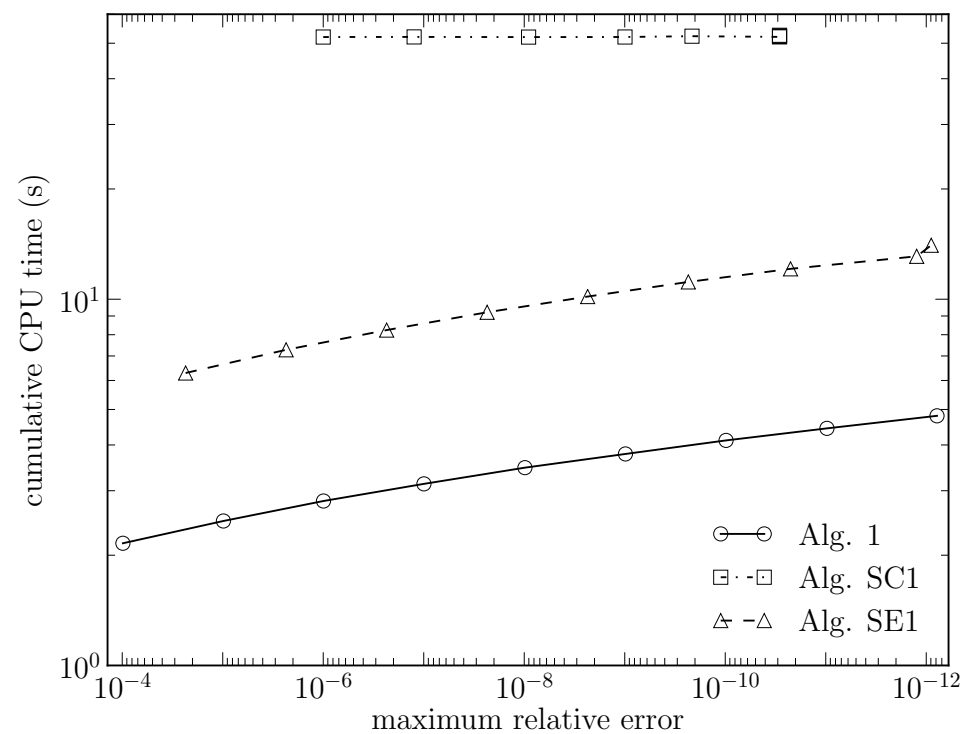

Fig. 11 Queueing MRM: cumulative CPU time in seconds required to compute $\operatorname{ETRR}\left(t_{n}\right), t_{n}=5 \mathrm{~h}, 10 \mathrm{~h}, \ldots, 10^{5} \mathrm{~h}$ as a function of the maximum over $t$ of the actual relative error in Algorithms 1, SC1, and SE1. (In each case, the left-most symbol corresponds to $\varepsilon=10^{-4}$.) 


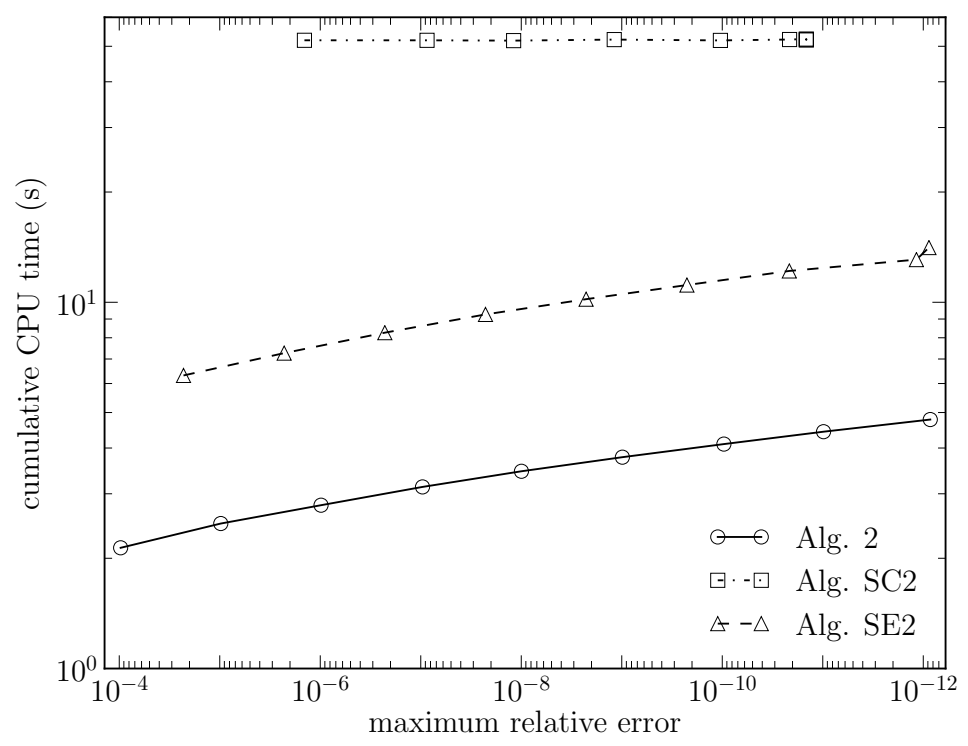

Fig. 12 Queueing MRM: cumulative CPU time in seconds required to compute $\operatorname{EARR}\left(t_{n}\right), t_{n}=5 \mathrm{~h}, 10 \mathrm{~h}, \ldots, 10^{5} \mathrm{~h}$, as a function of the maximum over $t_{n}$ of the actual relative error in Algorithms 2, SC2, and SE2. (In each case, the left-most symbol corresponds to $\varepsilon=10^{-4}$.)

new algorithms to be lower than that of the variants of the randomization method developed in (Suñé and Carrasco, 2005), which allow to compute the expected reward rates with control of the relative error, and lower than the run-time computational cost of the approach that consists in using iteratively the algorithms developed in (Sericola, 1999), which allow to compute the expected reward rates with control of the absolute error. The performance of the algorithms has been illustrated numerically, showing that the algorithms can be not only faster but also substantially more efficient than the alternatives in the sense of being able to achieve the same accuracy with a much lower run-time computational cost.

\section{Acknowledgments}

The author is indebted to Juan A. Carrasco for inspiring this investigation and to the referees, whose comments and suggestions have helped greatly to improve the paper.

\section{References}

Milton Abramowitz and Irene A Stegun. Handbook of mathematical functions: with formulas, graphs, and mathematical tables. Number 55 in Applied Mathematics Series. Courier Corporation, 1964. 10th printing (December 1972), with corrections.

Paul N Bowerman, Robert G Nolty, and Ernest M Scheuer. Calculation of the Poisson cumulative distribution function. IEEE Transactions on Reliability,, 39(2):158-161, 1990.

Juan A Carrasco. Solving dependability/performability irreducible Markov models using regenerative randomization. IEEE Transactions on Reliability, 52(3):319-329, 2003a. 
Juan Antonio Carrasco. Transient analysis of rewarded continuous time Markov models by regenerative randomization with Laplace transform inversion. The Computer Journal, 46(1):84-99, 2003b.

Juan Antonio Carrasco. Transient analysis of some rewarded Markov models using randomization with quasistationarity detection. IEEE Transactions on Computers, 53(9):1106-1120, 2004.

Juan Antonio Carrasco. Numerically stable methods for the computation of exit rates in Markov chains. Methodology and Computing in Applied Probability, 2015. To appear.

Peter M. Chen, Edward K. Lee, Garth A. Gibson, Randy H. Katz, and David A. Patterson. RAID: High-performance, reliable secondary storage. ACM Computing Surveys, 26(2):145-185, 1994.

K. P. Choi. On the median of gamma distributions and an equation of Ramanujan. Proc. Americal Mathematical Society, 121:245-251, 1994.

Laurent Fousse, Guillaume Hanrot, Vincent Lefèvre, Patrick Pélissier, and Paul Zimmermann. MPFR: A multiple-precision binary floating-point library with correct rounding. ACM Transactions on Mathematical Software, 33(2):13:1-13:15, June 2007.

Bennett L Fox and Peter W Glynn. Computing Poisson probabilities. Communications of the ACM, 31(4):440-445, 1988.

Peter Glynn. Upper bounds on Poisson tail probabilities. Operations Research Letters, 6(1):9-14, 1987.

Winfried K Grassmann. Transient solutions in Markovian queueing systems. Computers $\&$ Operations Research, 4(1):47-53, 1977.

Donald Gross and Douglas R Miller. The randomization technique as a modeling tool and solution procedure for transient Markov processes. Operations Research, 32(2):343-361, 1984.

IEEE754. IEEE standard for binary floating-point arithmetic. ANSI/IEEE Std 754-1985, 1985.

IEEE754-2008. IEEE standard for floating-point arithmetic - redline. IEEE Std 754-2008 (Revision of IEEE Std 754-1985) - Redline, 2008.

Massaki Kijima. Markov Processes for Stochastic Modeling. Chapman \& Hall, 1997.

L. Knüsel. Computation of the chi-square and Poisson distribution. SIAM Journal of Scientific and Statistical Computing, 7(3):1022-1036, 1986.

Takeshi Ogita, Siegfried M. Rump, and Shin'ichi Oishi. Accurate sum and dot product. SIAM Journal on Scientific Computing, 26(6):1955-1988, 2005.

Bruno Sericola. Availability analysis of repairable computer systems and stationarity detection. IEEE Transactions on Computers, 48(11):1166-1172, 1999.

Roger B Sidje, Kevin Burrage, and Shev MacNamara. Inexact uniformization method for computing transient distributions of Markov chains. SIAM Journal on Scientific Computing, 29(6):2562-2580, 2007.

Richard M. Stallman et al. Using the GNU Compiler Collection. For GCC version 4.7.2. Free Software Foundation, 2012.

Víctor Suñé and Juan A. Carrasco. Efficient implementations of the randomization method with control of the relative error. Computers \& Operations Research, 32:1089-1114, 2005.

Aad P. A. van Moorsel and William H. Sanders. Adaptive uniformization. Stochastic Models, 10(3): 619-647, 1994.

\section{A Proofs}

We will make use of Lemmas 1 and 2 given next.

Lemma 1 Let $0<\lambda^{\prime} \leq \lambda, l, m, n, 0 \leq l \leq m \leq n$, and $f(k), g(k) \geq 0$, with $g(k)$ uniformly upper bounded and strictly positive for some $k, k \geq n$. Then,

$$
\frac{\sum_{k=l}^{m} f(k) P_{k}(\lambda)}{\sum_{k=n}^{\infty} g(k) P_{k}(\lambda)} \leq \frac{\sum_{k=l}^{m} f(k) P_{k}\left(\lambda^{\prime}\right)}{\sum_{k=n}^{\infty} g(k) P_{k}\left(\lambda^{\prime}\right)}
$$


Proof Using Lemma 1 in (Suñé and Carrasco, 2005) with $w(k)=f(k), i=l, j=m, \lambda_{1}=\lambda, \lambda_{2}=\lambda^{\prime}$, and $x=\lambda / \lambda^{\prime}$,

$$
\left(\frac{\lambda^{\prime}}{\lambda}\right)^{m} \mathrm{e}^{\left(\lambda-\lambda^{\prime}\right)} \sum_{k=l}^{m} f(k) P_{k}(\lambda) \leq \sum_{k=l}^{m} f(k) P_{k}\left(\lambda^{\prime}\right) .
$$

Using again the lemma, now with $w(k)=g(k), i=n, j=\infty, \lambda_{1}=\lambda, \lambda_{2}=\lambda^{\prime}$, and $x=\lambda / \lambda^{\prime}$,

$$
\sum_{k=n}^{\infty} g(k) P_{k}\left(\lambda^{\prime}\right) \leq\left(\frac{\lambda^{\prime}}{\lambda}\right)^{n} \mathrm{e}^{\left(\lambda-\lambda^{\prime}\right)} \sum_{k=n}^{\infty} g(k) P_{k}(\lambda) .
$$

Combining (43), (44), recalling that, by assumption, $g(k)>0$ for some $k, k \geq n$, and noting that, for $n \geq m$, $\left(\lambda^{\prime} / \lambda\right)^{n-m} \leq 1$

$$
\begin{aligned}
\frac{\sum_{k=l}^{m} f(k) P_{k}(\lambda)}{\sum_{k=n}^{\infty} g(k) P_{k}(\lambda)} & \leq \frac{\sum_{k=l}^{n} f(k) P_{k}\left(\lambda^{\prime}\right)}{\left(\frac{\lambda^{\prime}}{\lambda}\right)^{m} \mathrm{e}^{\left(\lambda-\lambda^{\prime}\right)}} \frac{\left(\frac{\lambda^{\prime}}{\lambda}\right)^{n} \mathrm{e}^{\left(\lambda-\lambda^{\prime}\right)}}{\sum_{k=n}^{\infty} g(k) P_{k}\left(\lambda^{\prime}\right)} \\
& =\left(\frac{\lambda^{\prime}}{\lambda}\right)^{n-m} \frac{\sum_{k=l}^{m} f(k) P_{k}\left(\lambda^{\prime}\right)}{\sum_{k=n+1}^{\infty} g(k) P_{k}\left(\lambda^{\prime}\right)} \\
& \leq \frac{\sum_{k=l}^{m} f(k) P_{k}\left(\lambda^{\prime}\right)}{\sum_{k=n+1}^{\infty} g(k) P_{k}\left(\lambda^{\prime}\right)} .
\end{aligned}
$$

$\square$

Lemma 2 Assume $\lambda>0, n \geq 0$, and $r \geq n$. If $r>\lambda-2, m \geq 1$, and $m^{\prime} \geq 1$, then

$$
\begin{aligned}
\sum_{j=r}^{\infty}\left(1-\frac{n}{j+1}\right) P_{j}(\lambda) \leq\left(1-\left(\frac{\lambda}{r+2}\right)^{m}\right)^{-1} & \left(\sum_{j=r}^{r+m-1}\left(1-\frac{n}{j+1}\right) P_{j}(\lambda)\right. \\
& \left.+\left(\frac{\lambda}{r+2}\right)^{m} \frac{m}{\lambda}\left(1-\left(\frac{\lambda}{r+2}\right)^{m^{\prime}}\right)^{-1} \sum_{j=r+1}^{r+m^{\prime}} P_{j}(\lambda)\right) .
\end{aligned}
$$

Proof Using that, for $j \geq r$,

$$
P_{j+m+1}(\lambda)=\lambda^{m+1} \frac{j !}{(j+m+1) !} P_{j}(\lambda)=\lambda^{m+1} \frac{1}{j+1} \prod_{i=2}^{m+1} \frac{1}{j+i} P_{j}(\lambda) \leq \lambda^{m+1} \frac{1}{j+1}\left(\frac{1}{r+2}\right)^{m} P_{j}(\lambda),
$$

we can write

$$
\begin{aligned}
\sum_{j=r}^{\infty}\left(1-\frac{n}{j+1}\right) P_{j}(\lambda) & =\sum_{j=r}^{r+m-1}\left(1-\frac{n}{j+1}\right) P_{j}(\lambda)+\sum_{j=r+m}^{\infty}\left(1-\frac{n}{j+1}\right) P_{j}(\lambda) \\
& =\sum_{j=r}^{r+m-1}\left(1-\frac{n}{j+1}\right) P_{j}(\lambda)+\frac{1}{\lambda} \sum_{j=r+m}^{\infty}(j+1-n) P_{j+1}(\lambda) \\
& =\sum_{j=r}^{r+m-1}\left(1-\frac{n}{j+1}\right) P_{j}(\lambda)+\frac{1}{\lambda} \sum_{j=r}^{\infty}(j+m+1-n) P_{j+m+1}(\lambda) \\
& \leq \sum_{j=r}^{r+m-1}\left(1-\frac{n}{j+1}\right) P_{j}(\lambda)+\frac{1}{\lambda} \sum_{j=r}^{\infty}(j+m+1-n) \lambda^{m+1} \frac{1}{j+1}\left(\frac{1}{r+2}\right)^{m} P_{j}(\lambda) \\
& =\sum_{j=r}^{r+m-1}\left(1-\frac{n}{j+1}\right) P_{j}(\lambda)+\left(\frac{\lambda}{r+2}\right)^{m} \sum_{j=r}^{\infty}\left(1-\frac{n}{j+1}\right) P_{j}(\lambda) \\
& +\left(\frac{\lambda}{r+2}\right)^{m} \sum_{j=r}^{\infty} \frac{m}{j+1} P_{j}(\lambda) \\
= & \sum_{j=r}^{r+m-1}\left(1-\frac{n}{j+1}\right) P_{j}(\lambda)+\left(\frac{\lambda}{r+2}\right)^{m} \sum_{j=r}^{\infty}\left(1-\frac{n}{j+1}\right) P_{j}(\lambda)
\end{aligned}
$$




$$
+\left(\frac{\lambda}{r+2}\right)^{m} \frac{m}{\lambda} \sum_{j=r+1}^{\infty} P_{j}(\lambda) .
$$

Since $r>\lambda-2$, we can invoke Proposition 1 in (Glynn, 1987) with $n$ replaced by $r+1$ and $m$ replaced by $m^{\prime}$ to bound $\sum_{j=r+1}^{\infty} P_{j}(\lambda)$ from above, obtaining

$$
\sum_{j=r+1}^{\infty} P_{j}(\lambda) \leq\left(1-\left(\frac{\lambda}{r+2}\right)^{m^{\prime}}\right)^{-1} \sum_{j=r+1}^{r+m^{\prime}} P_{j}(\lambda) .
$$

Therefore,

$$
\begin{aligned}
\sum_{j=r}^{\infty}\left(1-\frac{n}{j+1}\right) P_{j}(\lambda) \leq & \sum_{j=r}^{r+m-1}\left(1-\frac{n}{j+1}\right) P_{j}(\lambda)+\left(\frac{\lambda}{r+2}\right)^{m} \sum_{j=r}^{\infty}\left(1-\frac{n}{j+1}\right) P_{j}(\lambda) \\
& +\left(\frac{\lambda}{r+2}\right)^{m} \frac{m}{\lambda}\left(1-\left(\frac{\lambda}{r+2}\right)^{m^{\prime}}\right)^{-1} \sum_{j=r+1}^{r+m^{\prime}} P_{j}(\lambda)
\end{aligned}
$$

and the result follows by solving for $\sum_{j=r}^{\infty}(1-n /(j+1)) P_{j}(\lambda)$.

Proof of Proposition 1. That the truncation parameter $R_{1}$ given by (9) is finite follows from

$$
\lim _{r \rightarrow \infty} \frac{\frac{1}{r+3-\Lambda t} \frac{r+3}{r+2}\left(r+2+\frac{\Lambda t}{r+3-\Lambda t}\right) P_{r+1}(\Lambda t)}{\sum_{j=k+1}^{r} P_{j}(\Lambda t)}=0
$$

and the fact that, as assumed, $\delta>0$. With regard to Inequality (10), it trivially holds in the case $\sum_{j=0}^{k} P_{j}(\Lambda t) \leq 0.9$. In the case $\sum_{j=0}^{k} P_{j}(\Lambda t)>0.9$, we clearly have, by (8),

$$
0 \leq \frac{\sum_{j=k+1}^{\infty} P_{j}(\Lambda t)-\sum_{j=k+1}^{R_{1}} P_{j}(\Lambda t)}{\sum_{j=k+1}^{\infty} P_{j}(\Lambda t)}=\frac{1-\sum_{j=0}^{k} P_{j}(\Lambda t)-\left[1-\sum_{j=0}^{k} P_{j}(\Lambda t)\right]^{1 \mathrm{~b}}}{1-\sum_{j=0}^{k} P_{j}(\Lambda t)} .
$$

Besides, since the median of a Poisson distribution with parameter $\Lambda t$ is nonsmaller than $\Lambda t-\log 2$ (Choi, 1994), we also have $k \geq \Lambda t-\log 2>\Lambda t-2$. Therefore, by (9), we have $R_{1} \geq k+1>\Lambda t-1$, which implies $R_{1}+1>\Lambda t-2$, and can then bound $\sum_{j=R_{1}+1}^{\infty} P_{j}(\Lambda t)$ from above by invoking Lemma 2 with $r=R_{1}+1, n=0, \lambda=\Lambda t, m=1$, and $m^{\prime}=1$. The result is:

$$
\sum_{j=R_{1}+1}^{\infty} P_{j}(\Lambda t) \leq\left(1-\frac{\Lambda t}{R_{1}+3}\right)^{-1}\left(P_{R_{1}+1}(\Lambda t)+\frac{\Lambda t}{R_{1}+3} \frac{1}{\Lambda t}\left(1-\frac{\Lambda t}{R_{1}+3}\right)^{-1} P_{R_{1}+2}(\Lambda t)\right) .
$$

Using then (8), (45), $P_{R_{1}+2}(\Lambda t)=\left(\Lambda t /\left(R_{1}+2\right)\right) P_{R_{1}+1}(\Lambda t)$, and $(9)$,

$$
\begin{aligned}
\frac{1-\sum_{j=0}^{k} P_{j}(\Lambda t)-\left[1-\sum_{j=0}^{k} P_{j}(\Lambda t)\right]^{\mathrm{lb}}}{1-\sum_{j=0}^{k} P_{j}(\Lambda t)} & =\frac{\sum_{j=k+1}^{\infty} P_{j}(\Lambda t)-\sum_{j=k+1}^{R_{1}} P_{j}(\Lambda t)}{\sum_{j=k+1}^{\infty} P_{j}(\Lambda t)} \\
& =\frac{\sum_{j=R_{1}+1}^{\infty} P_{j}(\Lambda t)}{\sum_{j=k+1}^{\infty} P_{j}(\Lambda t)} \\
& <\frac{\sum_{j=R_{1}+1}^{\infty} P_{j}(\Lambda t)}{\sum_{j=k+1}^{R_{1}} P_{j}(\Lambda t)} \\
& \leq \frac{\left(1-\frac{\Lambda t}{R_{1}+3}\right)^{-1}\left(P_{R_{1}+1}(\Lambda t)+\frac{\Lambda t}{R_{1}+3} \frac{1}{\Lambda t}\left(1-\frac{\Lambda t}{R_{1}+3}\right)^{-1} P_{R_{1}+2}(\Lambda t)\right)}{\sum_{j=k+1}^{R_{1}} P_{j}(\Lambda t)} \\
& =\frac{\frac{1}{R_{1}+3-\Lambda t} \frac{R_{1}+3}{R_{1}+2}\left(R_{1}+2+\frac{\Lambda t}{R_{1}+3-\Lambda t}\right) P_{R_{1}+1}(\Lambda t)}{\sum_{j=k+1}^{R_{1}} P_{j}(\Lambda t)} \\
& \leq \delta,
\end{aligned}
$$

which proves Inequality $(10)$ in the case $\sum_{j=0}^{k} P_{j}(\Lambda t)>0.9$ and concludes the proof of Proposition 1 . 
Proof of Proposition 2. From (13), (14), and (12), the definition of $K_{1}$ is seen to be equivalent to

$$
K_{1}=\min \left\{k \geq 0: M_{k}-m_{k} \leq 2 \frac{\sum_{j=0}^{k} v_{j} P_{j}(\Lambda t)}{1-\sum_{j=0}^{k} P_{j}(\Lambda t)} \varepsilon+\left(m_{k}+M_{k}\right)(\varepsilon(1-\delta)-\delta)\right\} .
$$

Then, since (6) $M_{k}>0, k \geq 0$, and, as assumed, $\delta<1$ and $\varepsilon>\delta /(1-\delta)$, we have $\left(m_{k}+M_{k}\right)(\varepsilon(1-\delta)-\delta)>0$, $k \geq 0$, which combined with, by (2),

$$
\lim _{k \rightarrow \infty}\left(M_{k}-m_{k}\right)=\lim _{k \rightarrow \infty}\left(\max _{i} c_{i}^{(k)}-\min _{i} c_{i}^{(k)}\right)=0,
$$

implies that $K_{1}$ is finite.

It remains to prove the inequality

$$
\left|\frac{\left.\operatorname{ETRR}\left(t^{\prime}\right)-\widehat{\operatorname{ETRR}}\left(t^{\prime}, K_{1}\right)\right)}{\widehat{\operatorname{ETRR}}\left(t^{\prime}, K_{1}\right)}\right| \leq \varepsilon .
$$

To that end, we will start by bounding $\left|\widehat{\operatorname{ETRR}}\left(t^{\prime}, K_{1}\right)\right|$ from below. Using (11), that, by (10), $\left[1-\sum_{j=0}^{K_{1}} P_{j}\left(\Lambda t^{\prime}\right)\right]^{\mathrm{lb}} \geq$ $(1-\delta)\left(1-\sum_{j=0}^{K_{1}} P_{j}\left(\Lambda t^{\prime}\right)\right)$, that, as assumed, $\delta<1$, and that (6) $M_{K_{1}}>0, k \geq 0$, we obtain

$$
\begin{aligned}
\left|\widehat{\operatorname{ETRR}}\left(t^{\prime}, K_{1}\right)\right| & =\left|r_{\max }\left(\sum_{j=0}^{K_{1}} v_{j} P_{j}\left(\Lambda t^{\prime}\right)+\frac{m_{K_{1}}+M_{K_{1}}}{2}\left[1-\sum_{j=0}^{K_{1}} P_{j}\left(\Lambda t^{\prime}\right)\right]^{\mathrm{lb}}\right)\right| \\
& =r_{\max }\left(\sum_{j=0}^{K_{1}} v_{j} P_{j}\left(\Lambda t^{\prime}\right)+\frac{m_{K_{1}}+M_{K_{1}}}{2}\left[1-\sum_{j=0}^{K_{1}} P_{j}\left(\Lambda t^{\prime}\right)\right]^{\mathrm{lb}}\right) \\
& \geq r_{\max }\left(\sum_{j=0}^{K_{1}} v_{j} P_{j}\left(\Lambda t^{\prime}\right)+\frac{m_{K_{1}}+M_{K_{1}}}{2}(1-\delta)\left(1-\sum_{j=0}^{K_{1}} P_{j}\left(\Lambda t^{\prime}\right)\right)\right) \\
& >0 .
\end{aligned}
$$

Now, by Lemma 1 with $l=0, m=K_{1}, f(k)=v_{k}, \lambda=\Lambda t, n=K_{1}+1, g(k)=1$, and $\lambda^{\prime}=\Lambda t^{\prime}$,

$$
\frac{\sum_{j=0}^{K_{1}} v_{j} P_{j}(\Lambda t)}{1-\sum_{j=0}^{K_{1}} P_{j}(\Lambda t)}=\frac{\sum_{j=0}^{K_{1}} v_{j} P_{j}(\Lambda t)}{\sum_{j=K_{1}+1}^{\infty} P_{j}(\Lambda t)} \leq \frac{\sum_{j=0}^{K_{1}} v_{j} P_{j}\left(\Lambda t^{\prime}\right)}{\sum_{j=K_{1}+1}^{\infty} P_{j}\left(\Lambda t^{\prime}\right)}=\frac{\sum_{j=0}^{K_{1}} v_{j} P_{j}\left(\Lambda t^{\prime}\right)}{1-\sum_{j=0}^{K_{1}} P_{j}\left(\Lambda t^{\prime}\right)} .
$$

Then, using (1), (11), (3), all with $k=K_{1}$, that, by (10), $1-\sum_{j=0}^{K_{1}} P_{j}\left(\Lambda t^{\prime}\right)-\left[1-\sum_{j=0}^{K_{1}} P_{j}\left(\Lambda t^{\prime}\right)\right]^{1 \mathrm{~b}} \leq \delta(1-$ $\left.\sum_{j=0}^{K_{1}} P_{j}\left(\Lambda t^{\prime}\right)\right),(46)$, and (49),

$$
\begin{aligned}
\frac{\left|\operatorname{ETRR}\left(t^{\prime}\right)-\widehat{\operatorname{ETRR}}\left(t^{\prime}, K_{1}\right)\right|}{r_{\max }}= & \left|\sum_{j=K_{1}+1}^{\infty} v^{(j)} P_{j}\left(\Lambda t^{\prime}\right)-\frac{m_{K_{1}}+M_{K_{1}}}{2}\left[1-\sum_{j=0}^{K_{1}} P_{j}\left(\Lambda t^{\prime}\right)\right]^{\mathrm{lb}}\right| \\
= & \mid \sum_{j=K_{1}+1}^{\infty} v^{(j)} P_{j}\left(\Lambda t^{\prime}\right)-\frac{m_{K_{1}}+M_{K_{1}}}{2}\left(1-\sum_{j=0}^{K_{1}} P_{j}\left(\Lambda t^{\prime}\right)\right) \\
& +\frac{m_{K_{1}}+M_{K_{1}}}{2}\left(1-\sum_{j=0}^{K_{1}} P_{j}\left(\Lambda t^{\prime}\right)-\left[1-\sum_{j=0}^{K_{1}} P_{j}\left(\Lambda t^{\prime}\right)\right]^{\mathrm{lb}}\right) \mid \\
= & \mid \sum_{j=K_{1}+1}^{\infty} v^{(j)} P_{j}\left(\Lambda t^{\prime}\right)-\frac{m_{K_{1}}+M_{K_{1}}}{2} \sum_{j=K_{1}+1}^{\infty} P_{j}\left(\Lambda t^{\prime}\right) \\
& +\frac{m_{K_{1}}+M_{K_{1}}}{2}\left(1-\sum_{j=0}^{K_{1}} P_{j}\left(\Lambda t^{\prime}\right)-\left[1-\sum_{j=0}^{K_{1}} P_{j}\left(\Lambda t^{\prime}\right)\right]^{\mathrm{lb}}\right) \mid \\
= & \mid \sum_{j=K_{1}+1}^{\infty}\left(v^{(j)}-\frac{m_{K_{1}}+M_{K_{1}}}{2}\right) P_{j}\left(\Lambda t^{\prime}\right) \\
& +\frac{m_{K_{1}}+M_{K_{1}}}{2}\left(1-\sum_{j=0}^{K_{1}} P_{j}\left(\Lambda t^{\prime}\right)-\left[1-\sum_{j=0}^{K_{1}} P_{j}\left(\Lambda t^{\prime}\right)\right]^{\mathrm{lb}}\right) \mid
\end{aligned}
$$




$$
\begin{aligned}
\leq & \sum_{j=K_{1}+1}^{\infty}\left|v^{(j)}-\frac{m_{K_{1}}+M_{K_{1}}}{2}\right| P_{j}\left(\Lambda t^{\prime}\right) \\
& +\frac{m_{K_{1}}+M_{K_{1}}}{2}\left(1-\sum_{j=0}^{K_{1}} P_{j}\left(\Lambda t^{\prime}\right)-\left[1-\sum_{j=0}^{K_{1}} P_{j}\left(\Lambda t^{\prime}\right)\right]^{\mathrm{lb}}\right) \\
\leq & \frac{M_{K_{1}}-m_{K_{1}}}{2} \sum_{j=K_{1}+1}^{\infty} P_{j}\left(\Lambda t^{\prime}\right)+\frac{m_{K_{1}}+M_{K_{1}}}{2} \delta\left(1-\sum_{j=0}^{K_{1}} P_{j}\left(\Lambda t^{\prime}\right)\right) \\
= & \left(\frac{M_{K_{1}}-m_{K_{1}}}{2}+\delta \frac{m_{K_{1}}+M_{K_{1}}}{2}\right)\left(1-\sum_{j=0}^{K_{1}} P_{j}\left(\Lambda t^{\prime}\right)\right) \\
\leq & \left(\frac{\sum_{j=0}^{K_{1}} v_{j} P_{j}(\Lambda t)}{1-\sum_{j=0}^{K_{1}} P_{j}(\Lambda t)} \varepsilon+\frac{m_{K_{1}}+M_{K_{1}}}{2}(\varepsilon(1-\delta)-\delta)\right. \\
& \left.+\delta \frac{m_{K_{1}}+M_{K_{1}}}{2}\right)\left(1-\sum_{j=0}^{K_{1}} P_{j}\left(\Lambda t^{\prime}\right)\right) \\
= & \left(\frac{\sum_{j=0}^{K_{1}} v_{j} P_{j}(\Lambda t)}{1-\sum_{j=0}^{K_{1}} P_{j}(\Lambda t)} \varepsilon+\frac{m_{K_{1}}+M_{K_{1}}}{2} \varepsilon(1-\delta)\right)\left(1-\sum_{j=0}^{K_{1}} P_{j}\left(\Lambda t^{\prime}\right)\right) \\
\leq & \left(\frac{\sum_{j=0}^{K_{1}} v_{j} P_{j}\left(\Lambda t^{\prime}\right)}{1-\sum_{j=0}^{K_{1}} P_{j}\left(\Lambda t^{\prime}\right)} \varepsilon+\frac{m_{K_{1}}+M_{K_{1}}}{2} \varepsilon(1-\delta)\right)\left(1-\sum_{j=0}^{K_{1}} P_{j}\left(\Lambda t^{\prime}\right)\right) \\
= & \sum_{j=0}^{K_{1}} v_{j} P_{j}\left(\Lambda t^{\prime}\right) \varepsilon+\frac{m_{K_{1}}+M_{K_{1}}}{2} \varepsilon(1-\delta)\left(1-\sum_{j=0}^{K_{1}} P_{j}\left(\Lambda t^{\prime}\right)\right) .
\end{aligned}
$$

Finally, combining (48), (50),

$$
\begin{aligned}
\left|\frac{\left.\operatorname{ETRR}\left(t^{\prime}\right)-\widehat{\operatorname{ETRR}}\left(t^{\prime}, K_{1}\right)\right)}{\widehat{\operatorname{ETRR}}\left(t^{\prime}, K_{1}\right)}\right| & \leq \frac{\sum_{j=0}^{K_{1}} v_{j} P_{j}\left(\Lambda t^{\prime}\right) \varepsilon+\frac{m_{K_{1}}+M_{K_{1}}}{2} \varepsilon(1-\delta)\left(1-\sum_{j=0}^{K_{1}} P_{j}\left(\Lambda t^{\prime}\right)\right)}{\sum_{j=0}^{K_{1}} v_{j} P_{j}\left(\Lambda t^{\prime}\right)+\frac{m_{K_{1}}+M_{K_{1}}}{2}(1-\delta)\left(1-\sum_{j=0}^{K_{1}} P_{j}\left(\Lambda t^{\prime}\right)\right)} \\
& =\varepsilon .
\end{aligned}
$$

This completes the proof of Proposition 2.

Proof of Proposition 3. That the truncation parameter $R_{2}$ given by (28) is finite follows from

$$
\lim _{r \rightarrow \infty} \frac{\frac{1}{r+3-\Lambda t} \frac{r+3}{r+2}\left(r+2+\frac{\Lambda t}{r+3-\Lambda t}\right) P_{r+1}(\Lambda t)}{\sum_{j=k+2}^{r} P_{j}(\Lambda t)}=0
$$

and the fact that, as assumed, $\delta>0$. Similarly, that the truncation parameter $R_{3}$ given by (30) is finite follows from

$$
\lim _{r \rightarrow \infty} \frac{\frac{1}{r+3-\Lambda t} \frac{r+3}{r+2}\left(r+1-k+\frac{\Lambda t}{r+3-\Lambda t}\right) P_{r+1}(\Lambda t)}{\sum_{j=k+1}^{r} \frac{j-k}{j+1} P_{j}(\Lambda t)}=0
$$

and the fact that $\delta>0$.

In the case $\sum_{j=0}^{k+1} P_{j}(\Lambda t) \leq 0.9$, Inequality (31) is trivially true. In the case $\sum_{j=0}^{k+1} P_{j}(\Lambda t)>0.9$, we have, by $(27)$,

$$
0 \leq \frac{\sum_{j=k+2}^{\infty} P_{j}(\Lambda t)-\sum_{j=k+2}^{R_{2}} P_{j}(\Lambda t)}{\sum_{j=k+2}^{\infty} P_{j}(\Lambda t)}=\frac{1-\sum_{j=0}^{k+1} P_{j}(\Lambda t)-\left[1-\sum_{j=0}^{k+1} P_{j}(\Lambda t)\right]^{\mathrm{lb}}}{1-\sum_{j=0}^{k+1} P_{j}(\Lambda t)} .
$$

Besides, we necessarily have $k+1 \geq \Lambda t-\log 2$ because the median of a Poisson distribution with parameter $\Lambda t$ is nonsmaller than $\Lambda t-\log 2$ (Choi, 1994). Therefore, by (28), we also have $R_{2} \geq k+2 \geq \Lambda t-\log 2+1>\Lambda t$, which implies $R_{2}+1>\Lambda t-2$, and can then bound $\sum_{j=R_{2}+1}^{\infty} P_{j}(\Lambda t)$ from above by invoking Lemma 2 with $r=R_{2}+1$, $n=0, \lambda=\Lambda t, m=1$, and $m^{\prime}=1$. The result is:

$$
\sum_{j=R_{2}+1}^{\infty} P_{j}(\Lambda t) \leq\left(1-\frac{\Lambda t}{R_{2}+3}\right)^{-1}\left(P_{R_{2}+1}(\Lambda t)+\frac{\Lambda t}{R_{2}+3} \frac{1}{\Lambda t}\left(1-\frac{\Lambda t}{R_{2}+3}\right)^{-1} P_{R_{2}+2}(\Lambda t)\right) .
$$


Then, using (27), (51), $P_{R_{2}+2}(\Lambda t)=\left(\Lambda t /\left(R_{2}+2\right)\right) P_{R_{2}+1}(\Lambda t)$, and (28),

$$
\begin{aligned}
\frac{1-\sum_{j=0}^{k+1} P_{j}(\Lambda t)-\left[1-\sum_{j=0}^{k+1} P_{j}(\Lambda t)\right]^{\mathrm{lb}}}{1-\sum_{j=0}^{k+1} P_{j}(\Lambda t)} & =\frac{\sum_{j=k+2}^{\infty} P_{j}(\Lambda t)-\sum_{j=k+2}^{R_{2}} P_{j}(\Lambda t)}{\sum_{j=k+2}^{\infty} P_{j}(\Lambda t)} \\
& =\frac{\sum_{j=R_{2}+1}^{\infty} P_{j}(\Lambda t)}{\sum_{j=k+2}^{\infty} P_{j}(\Lambda t)} \\
& <\frac{\sum_{j=R_{2}+1}^{\infty} P_{j}(\Lambda t)}{\sum_{j=k+2}^{R_{2}} P_{j}(\Lambda t)} \\
& \leq \frac{\left(1-\frac{\Lambda t}{R_{2}+3}\right)^{-1}\left(P_{R_{2}+1}(\Lambda t)+\frac{\Lambda t}{R_{2}+3} \frac{1}{\Lambda t}\left(1-\frac{\Lambda t}{R_{2}+3}\right)^{-1} P_{R_{2}+2}(\Lambda t)\right)}{\sum_{j=k+2}^{R_{2}} P_{j}(\Lambda t)} \\
& =\frac{\frac{1}{R_{2}+3-\Lambda t} \frac{R_{2}+3}{R_{2}+2}\left(R_{2}+2+\frac{\Lambda t}{R_{2}+3-\Lambda t}\right) P_{R_{2}+1}(\Lambda t)}{\sum_{j=k+2}^{R_{2}} P_{j}(\Lambda t)} \\
& \leq \delta,
\end{aligned}
$$

showing Inequality (31) in the case $\sum_{j=0}^{k+1} P_{j}(\Lambda t)>0.9$.

It remains to prove Inequality (32). If $k+1 \leq \Lambda t$, or $k+1>\Lambda t$ and $1-(0.9 \Lambda t /(k+1-\Lambda t)) P_{k+1}(\Lambda t) \leq$ $\sum_{j=0}^{k+1} P_{j}(\Lambda t) \leq 0.9$, the inequality is trivially true. Otherwise, i.e., if $k+1>\Lambda t$, and $1-(0.9 \Lambda t /(k+1-$ $\Lambda t)) P_{k+1}(\Lambda t)>\sum_{j=0}^{k+1}$ or $\sum_{j=0}^{k+1} P_{j}(\Lambda t)>0.9$, we clearly have, by (22), (29),

$$
\begin{aligned}
0 & \leq \frac{\sum_{j=k+1}^{\infty} \frac{j-k}{j+1} P_{j}(\Lambda t)-\sum_{j=k+1}^{R_{3}} \frac{j-k}{j+1} P_{j}(\Lambda t)}{\sum_{j=k+1}^{\infty} \frac{j-k}{j+1} P_{j}(\Lambda t)} \\
& =\frac{P_{k+1}(\Lambda t)+\frac{\Lambda t-(k+1)}{\Lambda t}\left(1-\sum_{j=0}^{k+1} P_{j}(\Lambda t)\right)-\left[\sum_{j=k+1}^{\infty} \frac{j-k}{j+1} P_{j}(\Lambda t)\right]^{\mathrm{lb}}}{P_{k+1}(\Lambda t)+\frac{\Lambda t-(k+1)}{\Lambda t}\left(1-\sum_{j=0}^{k+1} P_{j}(\Lambda t)\right)} .
\end{aligned}
$$

Besides, since, by (30), $R_{3} \geq k+1>\Lambda t$, implying $R_{3}+1>\Lambda t-2$, we can invoke Lemma 2 with $r=R_{3}+1$, $n=k+1, \lambda=\Lambda t, m=1$, and $m^{\prime}=1$, obtaining

$$
\begin{aligned}
\sum_{j=R_{3}+1}^{\infty}\left(1-\frac{k+1}{j+1}\right) P_{j}(\Lambda t) & \leq\left(1-\frac{\Lambda t}{R_{3}+3}\right)^{-1} \\
& \times\left(\left(1-\frac{k+1}{R_{3}+2}\right) P_{R_{3}+1}(\Lambda t)+\frac{\Lambda t}{R_{3}+3} \frac{1}{\Lambda t}\left(1-\frac{\Lambda t}{R_{3}+3}\right)^{-1} P_{R_{3}+2}(\Lambda t)\right) .
\end{aligned}
$$

Then, using (22), (29), (52), $P_{R_{3}+2}(\Lambda t)=\left(\Lambda t /\left(R_{3}+2\right)\right) P_{R_{3}+1}(\Lambda t)$, and (30),

$$
\begin{aligned}
\frac{P_{k+1}(\Lambda t)+\frac{\Lambda t-(k+1)}{\Lambda t}}{P_{k+1}(\Lambda t)+\frac{\Lambda t-(k+1)}{\Lambda t}\left(1-\sum_{j=0}^{k+1} P_{j}(\Lambda t)\right)} & \\
= & \frac{\sum_{j=k+1}^{\infty} \frac{j-k}{j+1} P_{j}(\Lambda t)-\sum_{j=k+1}^{R_{3}} \frac{j-k}{j+1} P_{j}(\Lambda t)}{\sum_{j=k+1}^{\infty} \frac{j-k}{j+1} P_{j}(\Lambda t)} \\
= & \frac{\sum_{j=R_{3}+1}^{\infty} \frac{j-k}{j+1} P_{j}(\Lambda t)}{\sum_{j=k+1}^{\infty} \frac{j-k}{j+1} P_{j}(\Lambda t)} \\
& <\frac{\sum_{j=R_{3}+1}^{\infty} \frac{j-k}{j+1} P_{j}(\Lambda t)}{\sum_{j=k+1}^{R_{3}} \frac{j-k}{j+1} P_{j}(\Lambda t)} \\
& =\frac{\sum_{j=R_{3}+1}^{\infty}\left(1-\frac{k+1}{j+1}\right) P_{j}(\Lambda t)}{\sum_{j=k+1}^{R_{3}} \frac{j-k}{j+1} P_{j}(\Lambda t)} \\
& \leq \frac{\left(1-\frac{\Lambda t}{R_{3}+3}\right)^{-1}\left(\left(1-\frac{k+1}{R_{3}+2}\right) P_{R_{3}+1}(\Lambda t)+\frac{\Lambda t}{R_{3}+3} \frac{1}{\Lambda t}\left(1-\frac{\Lambda t}{R_{3}+3}\right)^{-1} P_{R_{3}+2}(\Lambda t)\right)}{\sum_{j=k+1}^{R_{3}} \frac{j-k}{j+1} P_{j}(\Lambda t)}
\end{aligned}
$$




$$
\begin{aligned}
& \leq \frac{\frac{1}{R_{3}+3-\Lambda t} \frac{R_{3}+3}{R_{3}+2}\left(R_{3}+1-k+\frac{\Lambda t}{R_{3}+3-\Lambda t}\right) P_{R_{3}+1}(\Lambda t)}{\sum_{j=k+1}^{R_{3}} \frac{j-k}{j+1} P_{j}(\Lambda t)} \\
& \leq \delta,
\end{aligned}
$$

which proves Inequality (32) in the case $k+1>\Lambda t$, and $1-(0.9 \Lambda t /(k+1-\Lambda t)) P_{k+1}(\Lambda t)>\sum_{j=0}^{k+1}$ or $\sum_{j=0}^{k+1} P_{j}(\Lambda t)>$ 0.9 and concludes the proof of Proposition 3.

Proof of Proposition 4. From (35), (36), and (12), the definition of $K_{2}$ is readily seen to be equivalent to

$$
K_{2}=\min \left\{k \geq 0: M_{k}-m_{k} \leq 2 \frac{\sum_{j=0}^{k} w_{j} P_{j}(\Lambda t)}{P_{k+1}(\Lambda t)+\frac{\Lambda t-(k+1)}{\Lambda t}\left(1-\sum_{j=0}^{k+1} P_{j}(\Lambda t)\right)} \varepsilon+\left(m_{k}+M_{k}\right)(\varepsilon(1-\delta)-\delta)\right\} .
$$

Then, since (6) $M_{k}>0, k \geq 0$, and, as assumed, $\delta<1$ and $\varepsilon>\delta /(1-\delta)$, we have $\left(m_{k}+M_{k}\right)(\varepsilon(1-\delta)-\delta)>0$, $k \geq 0$, which combined with $(47) \lim _{k \rightarrow \infty}\left(M_{k}-m_{k}\right)=0$ implies that $K_{2}$ is finite.

To show the inequality

$$
\left|\frac{\operatorname{EARR}\left(t^{\prime}\right)-\widehat{\operatorname{EARR}}\left(t^{\prime}, K_{2}\right)}{\widehat{\operatorname{EARR}}\left(t^{\prime}, K_{2}\right)}\right| \leq \varepsilon
$$

we will start by obtaining a suitable lower bound for $\left|\widehat{\operatorname{EARR}}\left(t^{\prime}, K_{2}\right)\right| / r_{\max }$. We have, by (31),

$$
\left[1-\sum_{j=0}^{K_{2}+1} P_{j}\left(\Lambda t^{\prime}\right)\right]^{1 \mathrm{~b}} \geq(1-\delta)\left(1-\sum_{j=0}^{K_{2}+1} P_{j}\left(\Lambda t^{\prime}\right)\right)
$$

and, by (22), (32),

$$
\left[\sum_{j=K_{2}+1}^{\infty} \frac{j-K_{2}}{j+1} P_{j}\left(\Lambda t^{\prime}\right)\right]^{\mathrm{lb}} \geq(1-\delta)\left(\sum_{j=K_{2}+1}^{\infty} \frac{j-K_{2}}{j+1} P_{j}\left(\Lambda t^{\prime}\right)\right) .
$$

Then, using (34) and the fact that, as assumed, $\delta<1$,

$$
\begin{aligned}
\left|\frac{\widehat{\operatorname{EARR}}\left(t^{\prime}, K_{2}\right)}{r_{\max }}\right|= & \mid \sum_{j=0}^{K_{2}} w_{j} P_{j}\left(\Lambda t^{\prime}\right)+w_{K_{2}} \frac{K_{2}+1}{\Lambda t^{\prime}}\left[1-\sum_{j=0}^{K_{2}+1} P_{j}\left(\Lambda t^{\prime}\right)\right]^{\mathrm{lb}} \\
& +\frac{m_{K_{2}}+M_{K_{2}}}{2}\left[\sum_{j=K_{2}+1}^{\infty} \frac{j-K_{2}}{j+1} P_{j}\left(\Lambda t^{\prime}\right)\right]^{\mathrm{lb}} \mid \\
= & \sum_{j=0}^{K_{2}} w_{j} P_{j}\left(\Lambda t^{\prime}\right)+w_{K_{2}} \frac{K_{2}+1}{\Lambda t^{\prime}}\left[1-\sum_{j=0}^{K_{2}+1} P_{j}\left(\Lambda t^{\prime}\right)\right]^{\mathrm{lb}} \\
& +\frac{m_{K_{2}}+M_{K_{2}}}{2}\left[\sum_{j=K_{2}+1}^{\infty} \frac{j-K_{2}}{j+1} P_{j}\left(\Lambda t^{\prime}\right)\right]^{\mathrm{lb}} \\
\geq & \sum_{j=0}^{K_{2}} w_{j} P_{j}\left(\Lambda t^{\prime}\right)+w_{K_{2}} \frac{K_{2}+1}{\Lambda t^{\prime}}(1-\delta)\left(1-\sum_{j=0}^{K_{2}+1} P_{j}\left(\Lambda t^{\prime}\right)\right) \\
& +\frac{m_{K_{2}}+M_{K_{2}}}{2}(1-\delta) \sum_{j=K_{2}+1}^{\infty} \frac{j-K_{2}}{j+1} P_{j}\left(\Lambda t^{\prime}\right) \\
\triangleq & f\left(t^{\prime}, K_{2}\right) \\
> & 0 .
\end{aligned}
$$

Now, we will derive an upper bound for $\left|\operatorname{EARR}\left(t^{\prime}\right)-\widehat{\operatorname{EARR}}\left(t^{\prime}, K_{2}\right)\right| / r_{\max }$. To that end, we note that, given $k \geq 0$ and $j \geq k+1$, from (19) we obtain

$$
\begin{aligned}
w_{j} & =\frac{1}{j+1}\left(\sum_{l=0}^{k} v_{l}+\sum_{l=k+1}^{j} v_{l}\right) \\
& =\frac{1}{j+1}\left(\sum_{l=0}^{k} v_{l}+((j+1)-(k+1)) \frac{m_{k}+M_{k}}{2}+\sum_{l=k+1}^{j}\left(v_{l}-\frac{m_{k}+M_{k}}{2}\right)\right)
\end{aligned}
$$




$$
=\frac{k+1}{j+1} w_{k}+\frac{m_{k}+M_{k}}{2}-\frac{k+1}{j+1} \frac{m_{k}+M_{k}}{2}+\frac{1}{j+1} \sum_{l=k+1}^{j}\left(v_{l}-\frac{m_{k}+M_{k}}{2}\right) .
$$

Then, using (18), (34), (57), (22), and (3),

$$
\begin{aligned}
& \frac{\left|\operatorname{EARR}\left(t^{\prime}\right)-\widehat{\operatorname{EARR}}\left(t^{\prime}, k\right)\right|}{r_{\max }}=\mid \sum_{j=k+1}^{\infty} w_{j} P_{j}\left(\Lambda t^{\prime}\right)-\frac{k+1}{\Lambda t^{\prime}} w_{k}\left[1-\sum_{j=0}^{k+1} P_{j}\left(\Lambda t^{\prime}\right)\right]^{\mathrm{lb}} \\
& -\frac{m_{k}+M_{k}}{2}\left[\sum_{j=k+1}^{\infty} \frac{j-k}{j+1} P_{j}\left(\Lambda t^{\prime}\right)\right]^{1 \mathrm{~b}} \\
& =\mid \sum_{j=k+1}^{\infty} \frac{k+1}{j+1} w_{k} P_{j}\left(\Lambda t^{\prime}\right)+\frac{m_{k}+M_{k}}{2} \sum_{j=k+1}^{\infty} P_{j}\left(\Lambda t^{\prime}\right) \\
& -\sum_{j=k+1}^{\infty} \frac{k+1}{j+1} \frac{m_{k}+M_{k}}{2} P_{j}\left(\Lambda t^{\prime}\right) \\
& +\sum_{j=k+1}^{\infty} \frac{1}{j+1}\left(\sum_{l=k+1}^{j}\left(v_{l}-\frac{m_{k}+M_{k}}{2}\right)\right) P_{j}\left(\Lambda t^{\prime}\right) \\
& -\frac{k+1}{\Lambda t^{\prime}} w_{k}\left[1-\sum_{j=0}^{k+1} P_{j}\left(\Lambda t^{\prime}\right)\right]^{1 \mathrm{~b}}-\frac{m_{k}+M_{k}}{2}\left[\sum_{j=k+1}^{\infty} \frac{j-k}{j+1} P_{j}\left(\Lambda t^{\prime}\right)\right]^{\mathrm{lb}} \mid \\
& =\mid \frac{k+1}{\Lambda t^{\prime}} w_{k} \sum_{j=k+2}^{\infty} P_{j}\left(\Lambda t^{\prime}\right)+\frac{m_{k}+M_{k}}{2} \sum_{j=k+1}^{\infty} P_{j}\left(\Lambda t^{\prime}\right) \\
& -\frac{k+1}{\Lambda t^{\prime}} \frac{m_{k}+M_{k}}{2} \sum_{j=k+2}^{\infty} P_{j}\left(\Lambda t^{\prime}\right) \\
& +\sum_{j=k+1}^{\infty} \frac{1}{j+1}\left(\sum_{l=k+1}^{j}\left(v_{l}-\frac{m_{k}+M_{k}}{2}\right)\right) P_{j}\left(\Lambda t^{\prime}\right) \\
& -\frac{k+1}{\Lambda t^{\prime}} w_{k}\left[1-\sum_{j=0}^{k+1} P_{j}\left(\Lambda t^{\prime}\right)\right]^{\mathrm{lb}}-\frac{m_{k}+M_{k}}{2}\left[\sum_{j=k+1}^{\infty} \frac{j-k}{j+1} P_{j}\left(\Lambda t^{\prime}\right)\right]^{\mathrm{lb}} \\
& =\mid \frac{k+1}{\Lambda t^{\prime}} w_{k}\left(1-\sum_{j=0}^{k+1} P_{j}\left(\Lambda t^{\prime}\right)\right)+\frac{m_{k}+M_{k}}{2}\left(1-\sum_{j=0}^{k} P_{j}\left(\Lambda t^{\prime}\right)\right) \\
& -\frac{k+1}{\Lambda t^{\prime}} \frac{m_{k}+M_{k}}{2}\left(1-\sum_{j=0}^{k+1} P_{j}\left(\Lambda t^{\prime}\right)\right) \\
& +\sum_{j=k+1}^{\infty} \frac{1}{j+1}\left(\sum_{l=k+1}^{j}\left(v_{l}-\frac{m_{k}+M_{k}}{2}\right)\right) P_{j}\left(\Lambda t^{\prime}\right) \\
& -\frac{k+1}{\Lambda t^{\prime}} w_{k}\left[1-\sum_{j=0}^{k+1} P_{j}\left(\Lambda t^{\prime}\right)\right]^{\mathrm{lb}}-\frac{m_{k}+M_{k}}{2}\left[\sum_{j=k+1}^{\infty} \frac{j-k}{j+1} P_{j}\left(\Lambda t^{\prime}\right)\right]^{\mathrm{lb}} \\
& =\mid \frac{k+1}{\Lambda t^{\prime}} w_{k}\left(1-\sum_{j=0}^{k+1} P_{j}\left(\Lambda t^{\prime}\right)\right)+\frac{m_{k}+M_{k}}{2} \sum_{j=k+1}^{\infty} \frac{j-k}{j+1} P_{j}\left(\Lambda t^{\prime}\right) \\
& +\sum_{j=k+1}^{\infty} \frac{1}{j+1}\left(\sum_{l=k+1}^{j}\left(v_{l}-\frac{m_{k}+M_{k}}{2}\right)\right) P_{j}\left(\Lambda t^{\prime}\right) \\
& -\frac{k+1}{\Lambda t^{\prime}} w_{k}\left[1-\sum_{j=0}^{k+1} P_{j}\left(\Lambda t^{\prime}\right)\right]^{\mathrm{lb}}-\frac{m_{k}+M_{k}}{2}\left[\sum_{j=k+1}^{\infty} \frac{j-k}{j+1} P_{j}\left(\Lambda t^{\prime}\right)\right]^{\mathrm{lb}} \mid
\end{aligned}
$$




$$
\begin{aligned}
= & \frac{k+1}{\Lambda t^{\prime}} w_{k}\left(1-\sum_{j=0}^{k+1} P_{j}\left(\Lambda t^{\prime}\right)-\left[1-\sum_{j=0}^{k+1} P_{j}\left(\Lambda t^{\prime}\right)\right]^{\mathrm{lb}}\right) \\
& +\frac{m_{k}+M_{k}}{2}\left(\sum_{j=k+1}^{\infty} \frac{j-k}{j+1} P_{j}\left(\Lambda t^{\prime}\right)-\left[\sum_{j=k+1}^{\infty} \frac{j-k}{j+1} P_{j}\left(\Lambda t^{\prime}\right)\right]^{\mathrm{lb}}\right) \\
& +\sum_{j=k+1}^{\infty} \frac{1}{j+1}\left(\sum_{l=k+1}^{j}\left(v_{l}-\frac{m_{k}+M_{k}}{2}\right)\right) P_{j}\left(\Lambda t^{\prime}\right) \mid \\
\leq & \frac{k+1}{\Lambda t^{\prime}} w_{k}\left(1-\sum_{j=0}^{k+1} P_{j}\left(\Lambda t^{\prime}\right)-\left[1-\sum_{j=0}^{k+1} P_{j}\left(\Lambda t^{\prime}\right)\right]^{\mathrm{lb}}\right) \\
& +\frac{m_{k}+M_{k}}{2}\left(\sum_{j=k+1}^{\infty} \frac{j-k}{j+1} P_{j}\left(\Lambda t^{\prime}\right)-\left[\sum_{j=k+1}^{\infty} \frac{j-k}{j+1} P_{j}\left(\Lambda t^{\prime}\right)\right]^{\mathrm{lb}}\right) \\
& +\sum_{j=k+1}^{\infty} \frac{1}{j+1}\left(\sum_{l=k+1}^{j}\left|v_{l}-\frac{m_{k}+M_{k}}{2}\right|\right) P_{j}\left(\Lambda t^{\prime}\right) \\
\leq & \frac{k+1}{\Lambda t^{\prime}} w_{k}\left(1-\sum_{j=0}^{k+1} P_{j}\left(\Lambda t^{\prime}\right)-\left[1-\sum_{j=0}^{k+1} P_{j}\left(\Lambda t^{\prime}\right)\right]^{\mathrm{lb}}\right) \\
& +\frac{m_{k}+M_{k}}{2}\left(\sum_{j=k+1}^{\infty} \frac{j-k}{j+1} P_{j}\left(\Lambda t^{\prime}\right)-\left[\sum_{j=k+1}^{\infty} \frac{j-k}{j+1} P_{j}\left(\Lambda t^{\prime}\right)\right]^{\mathrm{lb}}\right) \\
& +\frac{M_{k}-m_{k}}{2} \sum_{j=k+1}^{\infty} \frac{j-k}{j+1} P_{j}\left(\Lambda t^{\prime}\right) .
\end{aligned}
$$

Now, replacing $k$ by $K_{2}$ in the above inequality and using that, by (31),

$$
1-\sum_{j=0}^{K_{2}+1} P_{j}\left(\Lambda t^{\prime}\right)-\left[1-\sum_{j=0}^{K_{2}+1} P_{j}\left(\Lambda t^{\prime}\right)\right]^{1 \mathrm{~b}} \leq \delta\left(1-\sum_{j=0}^{K_{2}+1} P_{j}\left(\Lambda t^{\prime}\right)\right),
$$

that, by (32), (22),

$$
\sum_{j=K_{2}+1}^{\infty} \frac{j-K_{2}}{j+1} P_{j}\left(\Lambda t^{\prime}\right)-\left[\sum_{j=K_{2}+1}^{\infty} \frac{j-K_{2}}{j+1} P_{j}\left(\Lambda t^{\prime}\right)\right]^{\mathrm{lb}} \leq \delta \sum_{j=K_{2}+1}^{\infty} \frac{j-K_{2}}{j+1} P_{j}\left(\Lambda t^{\prime}\right),
$$

Inequality (3) with $k$ replaced by $K_{2}$, that, from (35), (36), (22), and (12),

$$
M_{K_{2}}-m_{K_{2}} \leq 2 \frac{\sum_{j=0}^{K_{2}} w_{j} P_{j}(\Lambda t)}{\sum_{j=K_{2}+1}^{\infty} \frac{j-K_{2}}{j+1} P_{j}(\Lambda t)} \varepsilon+\left(m_{k}+M_{k}\right)(\varepsilon(1-\delta)-\delta),
$$

and that, as assumed, $\delta<\varepsilon(1-\delta)$, gives

$$
\begin{aligned}
\frac{\left|\operatorname{EARR}\left(t^{\prime}\right)-\widehat{\operatorname{EARR}}\left(t^{\prime}, K_{2}\right)\right|}{r_{\max } \leq} & \frac{K_{2}+1}{\Lambda t^{\prime}} w_{K_{2}}\left(1-\sum_{j=0}^{K_{2}+1} P_{j}\left(\Lambda t^{\prime}\right)-\left[1-\sum_{j=0}^{K_{2}+1} P_{j}\left(\Lambda t^{\prime}\right)\right]^{\mathrm{lb}}\right) \\
& +\frac{m_{K_{2}}+M_{K_{2}}}{2}\left(\sum_{j=K_{2}+1}^{\infty} \frac{j-K_{2}}{j+1} P_{j}\left(\Lambda t^{\prime}\right)-\left[\sum_{j=K_{2}+1}^{\infty} \frac{j-K_{2}}{j+1} P_{j}\left(\Lambda t^{\prime}\right)\right]^{1 \mathrm{~b}}\right) \\
& +\frac{M_{K_{2}}-m_{K_{2}}}{2} \sum_{j=K_{2}+1}^{\infty} \frac{j-K_{2}}{j+1} P_{j}\left(\Lambda t^{\prime}\right) \\
\leq & \frac{K_{2}+1}{\Lambda t^{\prime}} w_{K_{2}} \delta\left(1-\sum_{j=0}^{K_{2}+1} P_{j}\left(\Lambda t^{\prime}\right)\right)+\frac{m_{K_{2}}+M_{K_{2}}}{2} \delta \sum_{j=K_{2}+1}^{\infty} \frac{j-K_{2}}{j+1} P_{j}\left(\Lambda t^{\prime}\right) \\
& +\frac{m_{K_{2}}-M_{K_{2}}}{2} \sum_{j=K_{2}+1}^{\infty} \frac{j-K_{2}}{j+1} P_{j}\left(\Lambda t^{\prime}\right)
\end{aligned}
$$




$$
\begin{aligned}
\leq & \frac{K_{2}+1}{\Lambda t^{\prime}} w_{K_{2}} \delta\left(1-\sum_{j=0}^{K_{2}+1} P_{j}\left(\Lambda t^{\prime}\right)\right)+\frac{m_{K_{2}}+M_{K_{2}}}{2} \delta \sum_{j=K_{2}+1}^{\infty} \frac{j-K_{2}}{j+1} P_{j}\left(\Lambda t^{\prime}\right) \\
& +\frac{\sum_{j=0}^{K_{2}} w_{j} P_{j}(\Lambda t)}{\sum_{j=K_{2}+1}^{\infty} \frac{j-K_{2}}{j+1} P_{j}(\Lambda t)} \sum_{j=K_{2}+1}^{\infty} \frac{j-K_{2}}{j+1} P_{j}\left(\Lambda t^{\prime}\right) \varepsilon \\
& +\frac{m_{K_{2}}+M_{K_{2}}}{2}(\varepsilon(1-\delta)-\delta) \sum_{j=K_{2}+1}^{\infty} \frac{j-K_{2}}{j+1} P_{j}\left(\Lambda t^{\prime}\right) \\
= & \frac{K_{2}+1}{\Lambda t^{\prime}} w_{K_{2}} \delta\left(1-\sum_{j=0}^{K_{2}+1} P_{j}\left(\Lambda t^{\prime}\right)\right) \\
& +\frac{\sum_{j=0}^{K_{2}} w_{j} P_{j}(\Lambda t)}{\sum_{j=K_{2}+1}^{\infty} \frac{j-K_{2}}{j+1} P_{j}(\Lambda t)} \sum_{j=K_{2}+1}^{\infty} \frac{j-K_{2}}{j+1} P_{j}\left(\Lambda t^{\prime}\right) \varepsilon \\
& +\frac{m_{K_{2}}+M_{K_{2}}}{2}(1-\delta) \sum_{j=K_{2}+1}^{\infty} \frac{j-K_{2}}{j+1} P_{j}\left(\Lambda t^{\prime}\right) \varepsilon \\
\leq & \frac{K_{2}+1}{\Lambda t^{\prime}} w_{K_{2}} \varepsilon(1-\delta)\left(1-\sum_{j=0}^{K_{2}+1} P_{j}\left(\Lambda t^{\prime}\right)\right) \\
& +\frac{\sum_{j=0}^{K_{2}} w_{j} P_{j}(\Lambda t)}{\sum_{j=K_{2}+1}^{\infty} \frac{j-K_{2}}{j+1} P_{j}(\Lambda t)} \sum_{j=K_{2}+1}^{\infty} \frac{j-K_{2}}{j+1} P_{j}\left(\Lambda t^{\prime}\right) \varepsilon \\
& +\frac{m_{K_{2}}+M_{K_{2}}}{2}(1-\delta) \sum_{j=K_{2}+1}^{\infty} \frac{j-K_{2}}{j+1} P_{j}\left(\Lambda t^{\prime}\right) \varepsilon
\end{aligned}
$$

Now, by Lemma 1 with $l=0, m=K_{2}, f(k)=w_{k}, \lambda=\Lambda t, n=K_{2}+1, g(k)=\left(k-K_{2}\right) /(k+1)$, and $\lambda^{\prime}=\Lambda t^{\prime}$,

$$
\frac{\sum_{j=0}^{K_{2}} w_{j} P_{j}(\Lambda t)}{\sum_{j=K_{2}+1}^{\infty} \frac{j-K_{2}}{j+1} P_{j}(\Lambda t)} \leq \frac{\sum_{j=0}^{K_{2}} w_{j} P_{j}\left(\Lambda t^{\prime}\right)}{\sum_{j=K_{2}+1}^{\infty} \frac{j-K_{2}}{j+1} P_{j}\left(\Lambda t^{\prime}\right)} .
$$

Therefore, by (55) and (56), recalling (53),

$$
\begin{aligned}
\frac{\operatorname{EARR}\left(t^{\prime}\right)-\widehat{\operatorname{EARR}}\left(t^{\prime}, K_{2}\right) \mid}{r_{\max }} \leq & \frac{K_{2}+1}{\Lambda t^{\prime}} w_{K_{2}} \varepsilon(1-\delta)\left(1-\sum_{j=0}^{K_{2}+1} P_{j}\left(\Lambda t^{\prime}\right)\right) \\
& +\frac{\sum_{j=0}^{K_{2}} w_{j} P_{j}\left(\Lambda t^{\prime}\right)}{\sum_{j=K_{2}+1}^{\infty} \frac{j-K_{2}}{j+1} P_{j}\left(\Lambda t^{\prime}\right)} \sum_{j=K_{2}+1}^{\infty} \frac{j-K_{2}}{j+1} P_{j}\left(\Lambda t^{\prime}\right) \varepsilon \\
& +\frac{m_{K_{2}}+M_{K_{2}}}{2}(1-\delta) \sum_{j=K_{2}+1}^{\infty} \frac{j-K_{2}}{j+1} P_{j}\left(\Lambda t^{\prime}\right) \varepsilon \\
= & \frac{K_{2}+1}{\Lambda t^{\prime}} w_{K_{2}} \varepsilon(1-\delta)\left(1-\sum_{j=0}^{K_{2}+1} P_{j}\left(\Lambda t^{\prime}\right)\right)+\sum_{j=0}^{K_{2}} w_{j} P_{j}\left(\Lambda t^{\prime}\right) \varepsilon \\
& +\frac{m_{K_{2}}+M_{K_{2}}}{2}(1-\delta) \sum_{j=K_{2}+1}^{\infty} \frac{j-K_{2}}{j+1} P_{j}\left(\Lambda t^{\prime}\right) \varepsilon \\
= & \varepsilon f\left(t^{\prime}, K_{2}\right) .
\end{aligned}
$$

Combined with (53), Inequality (57) gives

$$
\left|\frac{\operatorname{EARR}\left(t^{\prime}\right)-\widehat{\operatorname{EARR}}\left(t^{\prime}, K_{2}\right)}{\widehat{\operatorname{EARR}}\left(t^{\prime}, K_{2}\right)}\right| \leq \frac{\varepsilon f\left(t^{\prime}, K_{2}\right)}{f\left(t^{\prime}, K_{2}\right)}=\varepsilon
$$

This concludes the proof of Proposition 4. 